\title{
Lack of redundancy between electrophysiological measures of long- range neuronal communication
}

\author{
Daniel Strahnen ${ }^{*}$, Sampath K. T. Kapanaiah", Alexei M. Bygrave ${ }^{2}$ and Dennis Kätzel ${ }^{1 *}$ (D)
}

\begin{abstract}
Background: Communication between brain areas has been implicated in a wide range of cognitive and emotive functions and is impaired in numerous mental disorders. In rodent models, various metrics have been used to quantify inter-regional neuronal communication. However, in individual studies, typically, only very few measures of coupling are reported and, hence, redundancy across such indicators is implicitly assumed.

Results: In order to test this assumption, we here comparatively assessed a broad range of directional and nondirectional metrics like coherence, Weighted Phase Lag Index (WPLI), phase-locking value (PLV), pairwise phase consistency (PPC), parametric and non-parametric Granger causality (GC), partial directed coherence (PDC), directed transfer function (DTF), spike-phase coupling (SPC), cross-regional phase-amplitude coupling, amplitude crosscorrelations and others. We applied these analyses to simultaneous field recordings from the prefrontal cortex and the ventral and dorsal hippocampus in the schizophrenia-related Gria1-knockout mouse model which displays a robust novelty-induced hyperconnectivity phenotype. Using the detectability of coupling deficits in Gria ${ }^{-1-}$ mice and bivariate correlations within animals as criteria, we found that across such measures, there is a considerable lack of functional redundancy. Except for three pairwise correlations - PLV with PPC, PDC with DTF and parametric with non-parametric Granger causality_almost none of the analysed metrics consistently co-varied with any of the other measures across the three connections and two genotypes analysed. Notable exceptions to this were the correlation of coherence with PPC and PLV that was found in most cases, and partial correspondence between these three measures and Granger causality. Perhaps most surprisingly, partial directed coherence and Granger causality - sometimes regarded as equivalent measures of directed influence-diverged profoundly. Also, amplitude cross-correlation, spike-phase coupling and theta-gamma phase-amplitude coupling each yielded distinct results compared to all other metrics.
\end{abstract}

Conclusions: Our analysis highlights the difficulty of quantifying real correlates of inter-regional information transfer, underscores the need to assess multiple coupling measures and provides some guidelines which metrics to choose for a comprehensive, yet non-redundant characterization of functional connectivity.

Keywords: Hippocampal-prefrontal coherence, wPLI, Phase-amplitude coupling, Phase-locking value, Pairwise phase-consistency, Spike-phase coupling, Granger causality, Partial directed coherence, Amplitude cross-correlation, Grial

\footnotetext{
*Correspondence: daniel.strahnen@uni-ulm.de; dennis.kaetzel@uni-ulm.de ${ }^{1}$ Institute of Applied Physiology, Ulm University, Albert-Einstein-Allee 11, 89081 Ulm, Germany

Full list of author information is available at the end of the article
}

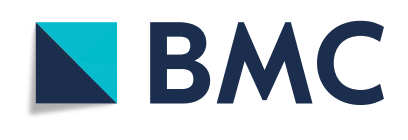

(c) The Author(s). 2021 Open Access This article is licensed under a Creative Commons Attribution 4.0 International License, which permits use, sharing, adaptation, distribution and reproduction in any medium or format, as long as you give appropriate credit to the original author(s) and the source, provide a link to the Creative Commons licence, and indicate if changes were made. The images or other third party material in this article are included in the article's Creative Commons licence, unless indicated otherwise in a credit line to the material. If material is not included in the article's Creative Commons licence and your intended use is not permitted by statutory regulation or exceeds the permitted use, you will need to obtain permission directly from the copyright holder. To view a copy of this licence, visit http://creativecommons.org/licenses/by/4.0/ The Creative Commons Public Domain Dedication waiver (http://creativecommons.org/publicdomain/zero/1.0/) applies to the data made available in this article, unless otherwise stated in a credit line to the data. 


\section{Background}

Communication between different brain regions is vital for cognition and emotion and is impaired in a variety of neurological and psychiatric disorders, including schizophrenia and depression. In order to better understand interregional communication in health and disease at the electrophysiological level in rodent models, local field potentials (LFPs) and sometimes action potentials (spikes) are typically recorded from two or more brain areas simultaneously in awake subjects. Subsequently, some measure of interdependency of the signals from two regions are computed (see Table 1 for an overview).

For example, an influential hypothesis known as communication through coherence (CTC) states that information exchange between two connected brain areas depends on the timing of the arrival of incoming activity in a specific phase of a certain network oscillation [25-28]. Therefore, coherence measures a synchrony of oscillations in a certain frequency range and with a certain phase shift that may allow the activity generated in one region to optimally affect the activity of another region.

In general, measures of phase synchronization aim to determine if two signals have a consistent phase relationship between each other. Despite being widely used, coherence is prone to confound by volume conduction $[22,29]$. Therefore, alternative measures of phase synchronization have been suggested. Nolte et al. demonstrated that using only the imaginary component of coherence $(\mathrm{ImC})$ effectively reduces the influence of a volume-conducted signal originating from a common source [3]. Alternatively, the Phase Lag Index (PLI) may be used, which disregards the magnitude of the phase lag between signals from two brain regions but evaluates if they differ from a symmetrical distribution [4]. The weighted PLI (wPLI), in turn, applies the combined advantages of the ImC and PLI by taking the detected phase lead or lag and weighing it by the magnitude of the $\operatorname{ImC}$ [5]. A constraint related to measures of phase synchronization like the ImC, PLI and wPLI is sample size bias, i.e. the observation of spurious non-zero synchrony even in the absence of real connections which increases with a lower number of samples [5]. Therefore Vinck et al. additionally introduced a debiassed estimator of the wPLI which is more independent from sample size and thus has a higher statistical power than previous measures [5]. For the sake of clarity, the debiassed wPLI will be referred to as wPLI throughout this study, and the stated older measures are not used.

It should be noted that several other metrics for phase synchronization exist, such as the phase-locking value (PLV) [6] and pairwise phase consistency (PPC) [7]. These measure the constancy of the difference between

Table 1 Common measures of synchrony and directionality in neuronal communication

\begin{tabular}{|c|c|c|c|c|}
\hline Category & Acronym & Metric & Description & References \\
\hline \multirow{6}{*}{$\begin{array}{l}\text { Non-directed } \\
\text { coupling, } \\
\text { synchrony }\end{array}$} & - & Coherence (magnitude) & Magnitude of the complex cross-spectrum & {$[1,2]$} \\
\hline & $\operatorname{ImC}$ & Imaginary part of coherence & Discards the real component of the cross-spectrum & {$[3]$} \\
\hline & PLI & Phase Lag Index & $\begin{array}{l}\text { Disregards the magnitude of the cross-spectrum and averages } \\
\text { the sign of phase differences }\end{array}$ & {$[4]$} \\
\hline & wPLI & Weighted phase lag index & $\begin{array}{l}\text { Phase lags are weighed by the magnitude of the imaginary } \\
\text { component of the cross-spectrum }\end{array}$ & {$[5]$} \\
\hline & PLV & Phase-locking value & Circular resultant vector length of the phase differences & {$[6]$} \\
\hline & PPC & Pairwise phase consistency & Computed based on the distribution of phase differences & {$[7]$} \\
\hline \multirow{2}{*}{$\begin{array}{l}\text { Directed (lead/lag, } \\
\text { LFP-based) }\end{array}$} & - & Coherence phase angle & Angle of the complex cross-spectrum & {$[8,9]$} \\
\hline & CC & $\begin{array}{l}\text { Cross-amplitude coupling, } \\
\text { amplitude cross-correlation }\end{array}$ & $\begin{array}{l}\text { Instantaneous amplitudes of two filtered LFPs are cross-correlated } \\
\text { and the lag at which the peak occurred is determined }\end{array}$ & {$[10]$} \\
\hline \multirow[t]{4}{*}{$\begin{array}{l}\text { Directed (causal } \\
\text { influence) }\end{array}$} & GC & Granger causality & $\begin{array}{l}\text { Quantifies if the past of one time series can predict the future of } \\
\text { another time series using autoregressive modelling }\end{array}$ & {$[11-14]$} \\
\hline & $\mathrm{npGC}$ & Non-parametric Granger causality & Granger causality based on spectral matrix factorization & {$[15]$} \\
\hline & PDC & Partial directed coherence & $\begin{array}{l}\text { Normalized metric based on GC that measures direct influence } \\
\text { from one time series to another }\end{array}$ & {$[16]$} \\
\hline & DTF & Direct transfer function & Adaptation to multiple input variables closely related to PDC & {$[17,18]$} \\
\hline \multirow{2}{*}{$\begin{array}{l}\text { Directed (phase- } \\
\text { locking of local } \\
\text { activity) }\end{array}$} & $S P C, M R L$ & $\begin{array}{l}\text { Spike-phase coupling, Mean } \\
\text { resultant vector length }\end{array}$ & $\begin{array}{l}\text { Circular concentration of the phase distribution at which spikes } \\
\text { occurred }\end{array}$ & {$[2,19-21]$} \\
\hline & $\begin{array}{l}\text { PAC, CFC, } \\
\mathrm{MI}\end{array}$ & $\begin{array}{l}\text { Phase amplitude coupling, cross- } \\
\text { frequency coupling, modulation } \\
\text { index }\end{array}$ & $\begin{array}{l}\text { Modulation of the amplitude of high-frequency oscillations in } \\
\text { one area by the phase of low-frequency oscillations from another } \\
\text { area }\end{array}$ & {$[22-24]$} \\
\hline \multirow{2}{*}{$\begin{array}{l}\text { Directed (lead lag, } \\
\text { spike-based) }\end{array}$} & - & Phase angle of MRL & Mean phase at which spikes occurred & {$[20]$} \\
\hline & - & Phase-shifted MRL & Calculation of the MRL based on phases at shifted lags & {$[2,19]$} \\
\hline
\end{tabular}


the instantaneous phases of two signals obtained either by applying Hilbert, wavelet or Fourier transformation and quantifying the distribution of phase differences either by taking the vector average or by determining the distribution of phase differences across observations, respectively. While PPC and PLV are very similar measures, the main advantage of the PPC metric is that it is not biassed by sample size and therefore more suitable for comparing datasets with varying sample size as reviewed in [30].

The stated measures of phase synchronization are attempts to quantify non-directed connectivity. This means that the quantification of coupling is essentially based on correlation analysis, ignores its temporal structure and assumes no direction of the influence from one region to another [30, 31]. However, LFP data can also be used to measure effective or directed connectivity [31]. These are parameters that quantify the potentially causal influence that the activity in one region exerts on another region by taking recurring pairwise patterns in the time series obtained from both regions into account. A computationally simple measure to detect directionality between two time series is cross-correlation. That means that correlations are calculated as the LFP signals are incrementally shifted against one another to obtain a cross-correlation as function of temporal shifts (lags). Adhikari et al. developed a method termed amplitude cross-correlation or cross-amplitude coupling in which the instantaneous amplitudes of two oscillatory signals filtered in a certain frequency range are cross-correlated to determine if one is leading or lagging the other [10]. If the lags at which the peak of the amplitude crosscorrelation function occurs are significantly different from $0 \mathrm{~ms}$, it is indicated that one region leads the other one with a certain consistency, which could be due to a directional influence from the leading onto the lagging region. This method was able to identify directional connectivity in the brain related to working memory and fear processing [32-34].

A different measure of directed influence is Granger causality (GC). It aims to infer causation based on the notion that one signal is helpful in predicting the other. In parametric GC, two separate autoregressive models (ARMs) are calculated and statistically compared: a univariate ARM, where the signal is predicted by a weighted combination of its own past values, and a bivariate ARM where the signal is additionally predicted by the second signal. If the inclusion of the bivariate AR leads to a reduction of variance of the predicted signal, one signal is said to Granger-cause the other [11]. GC can also be computed with non-parametric methods where the same information is obtained by first calculating the crossspectral density matrix and then applying Wilson's spectral matrix factorization as input to the GC algorithm; this approach has been demonstrated to be equivalent to parametric GC [35]. The mathematical foundations of GC and its application to neuroscience have been reviewed extensively elsewhere [11-14]. Related measures that can either be based on multivariate autoregressive models or on non-parametric methods for directionality estimation and allow analysis of more than two channels include the directed transfer function (DTF) [17] and partial directed coherence (PDC) [16]; see [18] for a review.

Other indicators of inter-regional communication that partly circumvent problems caused by volume conductance and are typically interpreted as indicating a causal directional influence include those that measure different types of neuronal activity in the different regions, i.e. a low-frequency LFP oscillation (usually in the theta-range) in the presumed dominating region and a local and high-frequency activity at the receiving end. In contrast to the metrics introduced before, historically, such measures were introduced by way of an actual biological discovery of such coupling phenomena, rather than by a priori mathematical considerations on how to best assess inter-regional communication. One option is to quantify the extent to which oscillations of distinct frequencies are coupled to each other, a phenomenon called crossfrequency coupling (CFC, [36]). Particularly, local phaseamplitude coupling (PAC) - the statistical relationship between the phase of a low-frequency and the amplitude of a high-frequency component-plays an important role in memory processing in the hippocampus of rats $[37,38]$ and humans [39]. However, cross-regional PAC between the hippocampus and prefrontal cortex has also been used and was associated with directed information flow and cognitive functions [22-24, 40]. Since high-frequency brain oscillations mainly reflect local aspects of information processing and low-frequency brain rhythms are relevant for inter-regional communication, CFC might represent a mechanism of transferring information from large-scale neuronal networks to local processes [36, 41].

Another widely used measure is based on the recording of spikes in one (potentially the influenced) region alongside the LFP in another (potentially the influencing) region. Spikes are generally not considered to be confounded by volume conductance or referencing, and they represent a more direct readout of the actual neuronal activity of a region. Phase-locking of neuronal firing to theta frequency hippocampal oscillations was shown for example in the prefrontal cortex (PFC) $[1,19]$, entorhinal cortex [42] and the amygdala [43]. For example, action potentials in these brain regions occurred rhythmically at the same phase of the hippocampal theta rhythm. Such spike-phase coupling (SPC) was observed to correlate with performance in multiple cognitive tasks $[1,19,44]$ and has been used to evaluate coupling deficits in mouse models related to schizophrenia $[2,20,45]$. 
The above-mentioned measures have been widely used for two decades to assess inter-regional neuronal communication in rodents during a variety of cognitive tasks and disease-related manipulations, mostly involving recordings from the hippocampus and prefrontal cortex $[1,2,20,21]$, but also increasingly from the thalamus [46] and the amygdala [47]. However, typically, only one or two measures of coupling are calculated and interpreted as sufficient surrogate to quantify task- or manipulation-related differences in actual information exchange between the analysed regions. In this analytical set-up, the contingency of the achieved conclusions on the choice of the coupling measure is usually not evaluated, but the redundancy of the various measures is implicitly assumed. This assumption is not justified, however, given the mathematical and partly biological differences between these constructs. Likewise, the dependence of the conclusions on the exact placements of electrodes within the analysed regions and the choice of reference are often not evaluated either. This presents a problem especially when interpreting negative data, i.e. the supposed absence of differences in coupling.

We therefore sought to evaluate the redundancy and contingencies of such coupling metrics. To this end, we recorded data during a simple behavioural assay-novelty-induced locomotion and its habituation over timein $\mathrm{Gria1}^{-/-}$(KO, knockout) mice and their littermate controls. We have recently shown that the Gria1-KO model, which recapitulates some behavioural deficits relevant to schizophrenia, shows profound and statedependent aberrations of hippocampal-prefrontal coupling in this task [48]. We focused on the most widely used connectivity measures-coherence magnitude and phase angle, wPLI, PPC, PLV, cross-amplitude coupling, parametric and non-parametric GC, PDC, DTF, cross-regional PAC, SPC and SPC-related directionality-with respect to three litmus tests' for redundancy: (a) detection of KO-related alterations of coupling across the $10 \mathrm{~min}$ test, (b) detection of KO-related changes of a measure over time and (c) bivariate withinanimal correlation of the analysed measures. We investigated connectivity between the medial prefrontal cortex (PFC) and the hippocampus-both the dorsal $(\mathrm{dHC})$ and the ventral $(\mathrm{vHC})$ partition. For the majority of the analysis, four commonly used frequency bands, delta $(\delta, 1-4 \mathrm{~Hz})$, theta $(\theta, 5-12$ $\mathrm{Hz})$, beta $(\beta, 15-30 \mathrm{~Hz})$ and low gamma $(\gamma, 30-48 \mathrm{~Hz})$ are distinguished, whereby the analysis of theta and gamma may be regarded as particularly informative due to the existence of spectral peaks indicating real underlying oscillatory processes.

\section{Results}

Elevated locomotor activity in Gria1-KO mice during measurement of interregional communication

In order to measure inter-regional coupling, we implanted 15 adult $\mathrm{Grial}^{-/-}$mice and 12 littermate controls unilaterally with LFP electrodes in 4 regions, PFC (2 electrodes), mediodorsal thalamus (MD, 1 electrode), dHC (1 electrode) and vHC (2 electrodes), and inserted screws for ground and reference above the cerebellum and frontal cortex, respectively (Fig. 1a). Recordings from all sites were made during a 10-min test of novelty-induced locomotor activity which confirmed the strongly elevated behavioural activity and failure of its short-term habituation over time in $\mathrm{Grial}^{-/-}$mice, as observed before (Fig. 1b, c [48]). After the experiments were completed, the placement of electrodes was evaluated through electrolytic lesion sites, and misplaced electrodes were excluded from the dataset; data from the MD was disregarded for most of the subsequent analysis because of the low number of animals with accurate placements. In accordance with our previous study in this mouse line [48], we recorded and analysed all data as referenced to the ground screw above the cerebellum by default and used the data from the frontal reference screw for a separate analysis (displayed in Fig. 7). We extracted LFP signals (Fig. 1d, e) from all depth electrodes and multi-unit activity (MUA) spikes from the prefrontal wires. For PAC, amplitude cross-correlations and SPC, the theta phase angle was extracted using a Hilbert transform or linear interpolation between consecutive cycles (Fig. 1f, g). Additionally, we sorted the LFP power values obtained from each electrode in distinct frequency bands according to the placement of electrodes in different subdivisions of the PFC (PrL, Cg1 and Cg2), $\mathrm{dHC}$ (apical dendritic layers of CA1, CA1 pyramidal cells, CA1 stratum oriens) and $\mathrm{vHC}$ (apical dendritic layers of CA1/CA3, CA1 pyramidal cells, dentate gyrus). While we did not conduct statistical analysis given the much smaller number of sites outside the target region (PrL in PFC and apical dendritic layers, including fissure, in the hippocampus), a qualitative inspection suggested that the placements inferred from lesion sites did not noticeably alter the obtained spectral LFP power (Fig. 1h-j).

\section{Differences in detecting delta and gamma-range coupling in Gria1-KO mice across measures of synchrony}

We first analysed phase synchronization along the two prefrontal-hippocampal connections (PFC-dHC and PFC-vHC) and within the hippocampus (vHC-dHC) using coherence, wPLI, PLV and PPC (Fig. 2a-r). We confirmed our previous observation [48] that PFC-dHC theta coherence is strongly elevated in Gria1-knockouts in a novel environment and further increases with time, mirroring the spatial exploration behaviour of this genotype (Fig. 1b, c, Fig. 2a, d). However, this phenotype was by no means specific to the PFC-dHC coupling, but also re-appeared in the PFC-vHC and vHC-dHC connections suggesting a broader deficit of excessive theta-range 


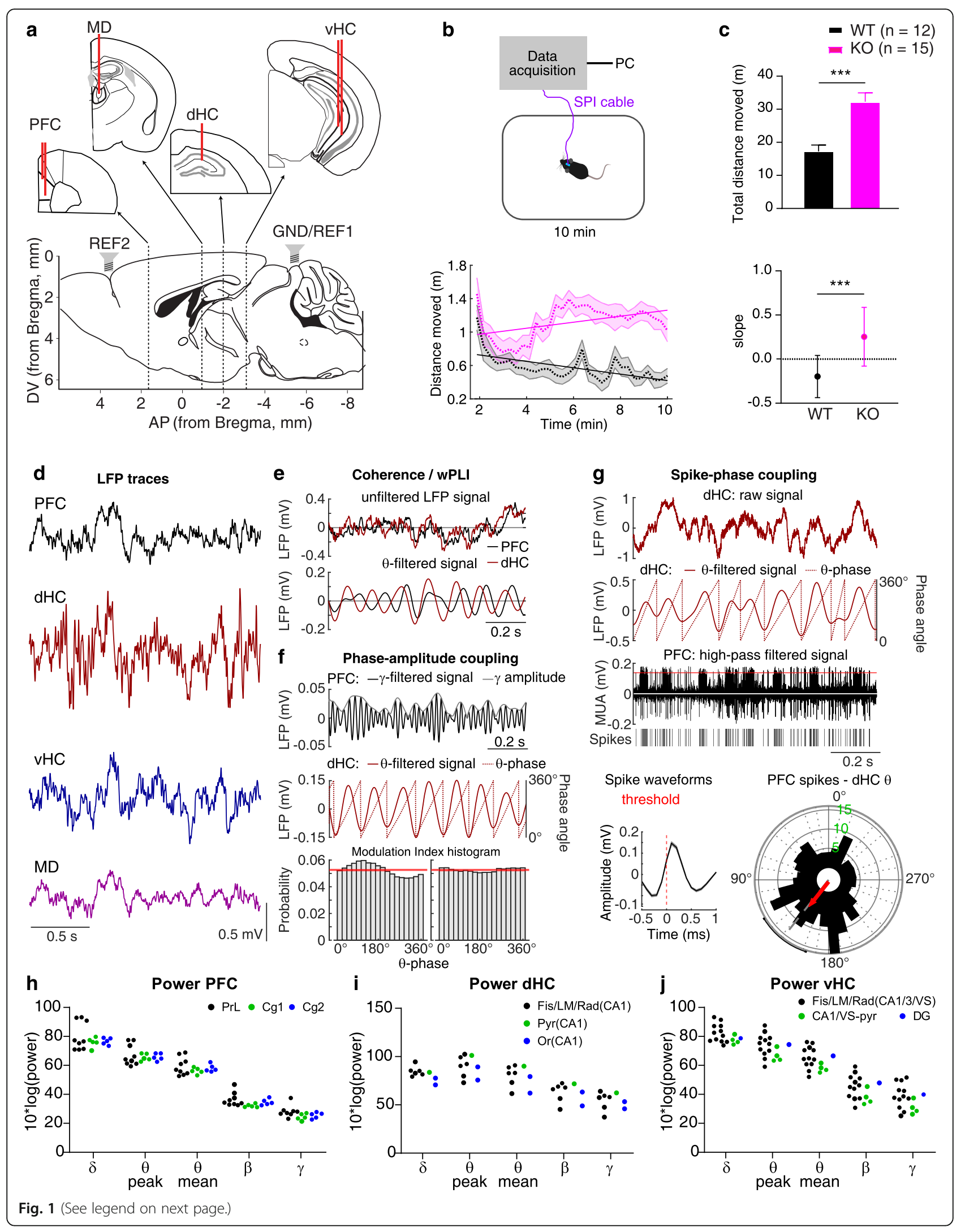


(See figure on previous page.)

Fig. 1 Experimental set-up, behaviour and recorded signals. a Placement of LFP and screw electrodes. b Top, experimental set-up; bottom, distance moved in $20 \mathrm{~s}$ bins by Gria ${ }^{-1-}$ (KO, purple) and wild-type controls (WT, black); dashed line indicating mean; shaded region representing SEM; solid overlaid line representing linear interpolations across time. $\mathbf{c}$ The same data as in $\mathbf{b}$ but displayed as total distance moved in 10 min (top) and slope of the interpolated line (bottom). ${ }^{* *} p<0.001, t$ test. $\mathbf{d}$ Examples of unfiltered LFP traces recorded in the four brain regions. $\mathbf{e}$ Illustration of the processing for connectivity measures using the same LFP frequency band in both regions; raw LFP signal (top) and LFP signal filtered in a specific frequency range (bottom). $\mathbf{f}$ Illustration of cross-regional $\theta-\gamma$ PAC, whereby the signal in one region is filtered in the low- $\gamma$ range and the amplitude is extracted (top), while the signal in the other region is filtered in the $\theta$ range and Hilbert-transformed to extract the $\theta$ phase (middle). The coupling strength is derived as a modulation index (MI) measuring the phase-related change of $\gamma$ amplitude (bottom). $\mathbf{g}$ Illustration of SPC; the hippocampal LFP (top) is filtered in the theta range and the instantaneous phase angle is extracted by linear interpolation (below, brown); the prefrontal high-pass filtered signal reveals MUA from which spikes are extracted by thresholding (below, single spikes, and bottom left, average of all extracted PFC spikes, black). A circular histogram is computed by assigning each spike to its theta phase angle, and the average of all vectors is calculated as mean resultant vector (red) whose length (MRL) is taken as indicator of SPC strength (bottom right). $\mathbf{h}-\mathbf{j}$ Power of LFP in the indicated frequency bands ( $x$-axis) and region (top of panel) displayed for each individual electrode that contributed to the WT dataset colour-coded by the sub-division in which it was placed; hippocampal layers: pyramidal (Pyr), stratum oriens (Or), lacunosummoleculare (LM), radiatum (Rad), and fissure (Fis). No statistical analysis was done given that rare placements

connectivity (Fig. 2b, c, e, f). Reassuringly, the same phenotype was revealed by the wPLI, PLV and PPC metric across connections (Fig. 2g-r). However, when inspecting the other frequency bands, findings were not particularly consistent between wPLI and the other three measures (which appeared very similar to each other). While all indicators revealed a reduced gamma-range PFC-dHC coupling in knockouts, a sole analysis with wPLI suggested further differences in the delta (PFC$\mathrm{dHC}, \mathrm{vHC}-\mathrm{dHC})$ and gamma (PFC-vHC) ranges that would have gone undetected, if using the other metrics (Fig. 2d-f, j-r). Also, qualitatively, wPLI resulted in spectra with a quite different shape compared to the other ones.

\section{Differences in detecting elevated inter-regional theta- range coupling in Gria1-KO mice across measures of directional communication}

An analysis of directional connectivity with parametric GC revealed a confirmatory but much more fine-grained picture with KO-induced aberrations in all four frequency bands depending on the connection and direction (Fig. 3a-c). Most prominently, we found strongly elevated theta range GC in knockouts for all projections departing in either subdivision of the hippocampus. This confirms the hippocampal (as opposed to prefrontal) origin of the theta hyper-connectivity phenotype in Gria1knockout mice that we had postulated before based on the normalization of this phenotype in mice with hippocampal rescue of GluA1 expression [48]. Likewise, beta/ gamma $\mathrm{dHC} \rightarrow \mathrm{PFC}$ GC was strongly reduced in knockouts (Fig. 3a), in line with reduced phase synchronization measures (Fig. 2d, j, m, p), while $\mathrm{PFC} \rightarrow \mathrm{dHC}$ beta and gamma GC were even mildly elevated. This again suggests a hippocampal origin of the observed reduced synchrony in this frequency range. The most prominent GC was found in the delta range, with $\mathrm{PFC} \rightarrow \mathrm{d} / \mathrm{vHC} \mathrm{GC}$ being significantly larger than the delta GC in the opposite direction in both genotypes.
Further, genotype-related differences in $\mathrm{vHC} \rightarrow \mathrm{PFC}$ and $\mathrm{dHC} \rightarrow \mathrm{vHC}$ delta GC were found that do not match with the results from the non-directional synchrony metrics (Fig. 2).

In contrast to $\mathrm{GC}$, significantly elevated theta $P D C$ in knockouts was only detected in the $\mathrm{dHC} \rightarrow \mathrm{PFC} / \mathrm{vHC}$ connections, but not in the $\mathrm{vHC} \rightarrow \mathrm{PFC} / \mathrm{dHC}$ projections. And in the beta/gamma ranges, there were virtually no matches between PDC and GC at all regarding genotype-related differences (except for a minority of null-results and trends; Fig. 3a-f). Assessing SPC using the mean resultant vector length (MRL) of the vector representing average spike occurrence in theta phase space [21], we found the opposite of what would have been assumed from the PDC metric: locking of PFC spikes to $v H C$ theta was higher in Gria1-knockouts, but phase-locking of PFC spikes to $d H C$ theta showed no difference between genotypes (the latter also contrasts with GC and all synchrony measures; Fig. 3g).

Further discrepancies appeared when analysing consistent phase differences (leads and lags) between potentially coupled oscillations in different regions to assess directionality. We investigated two directional measures obtainable from the SPC: the average theta phase of the MRL [20] and analysis of the effect of incremental shifts of the MUA relative to the theta cycle on the MRL [19]. The MRLs of PFC spikes relative to the dHC-but not vHC-theta phase were significantly shifted between genotypes: while they occurred during the rising phase of theta in knockouts, they occurred at its through in wildtype mice (Fig. 3h). Leading of PFC spikes relative to $\mathrm{dHC}$ and $\mathrm{vHC}$ theta was seen with phase-shifted MRL analysis in knockouts, but no significant difference between genotypes was detectable in this metric (Fig. 3i). The equivalent analysis, but conducted with PFC LFP (instead of spikes) using cross-amplitude coupling showed the opposite, namely a lead of $\mathrm{dHC}$ and $\mathrm{vHC}$ theta relative to prefrontal theta in knockouts, and differences between genotypes in both connections (Fig. 3j). 


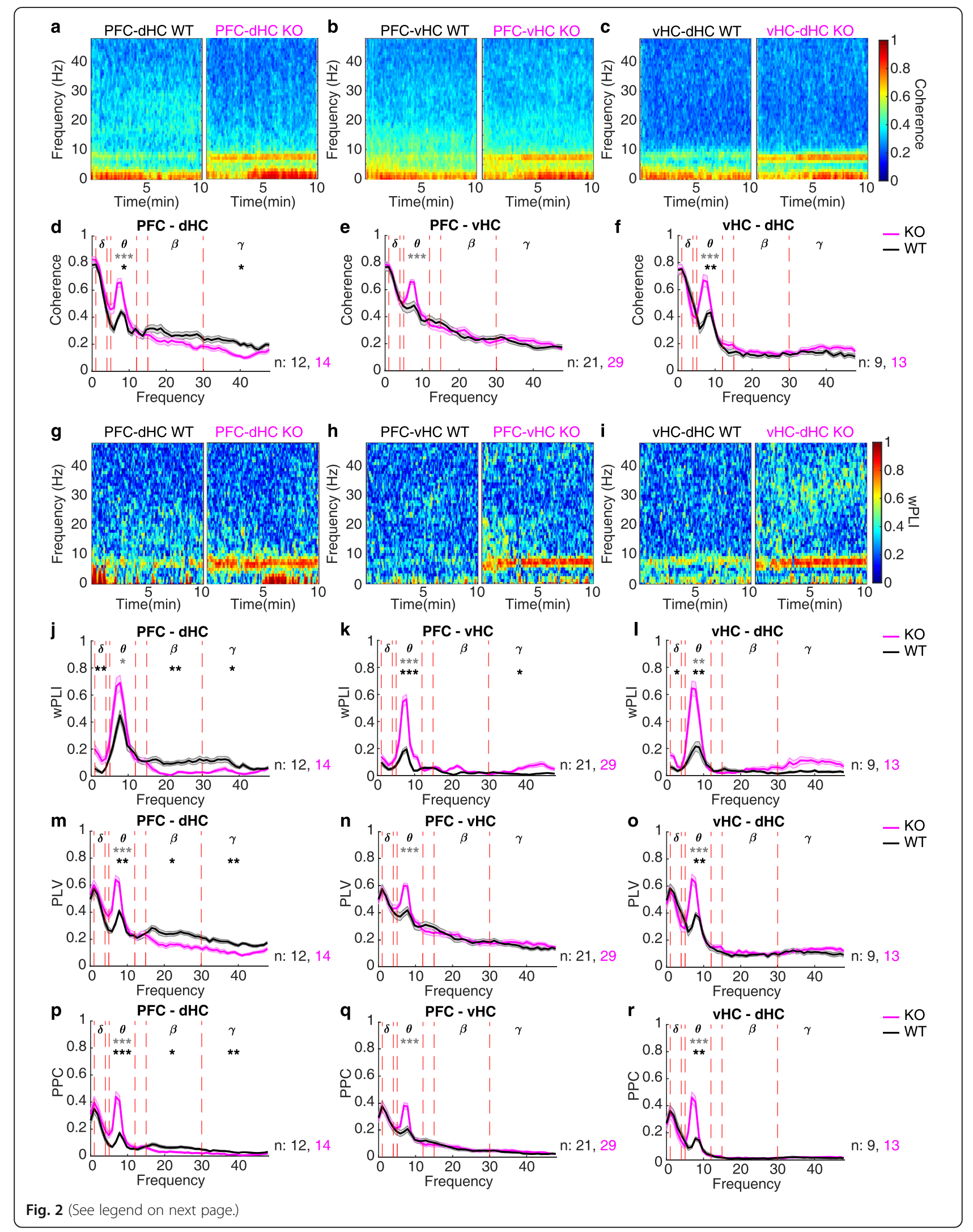


(See figure on previous page.)

Fig. 2 Non-directional measures of synchrony in $\mathrm{Gria}^{-1-}$ and wild-type controls across 10 min novelty-induced activity. a-I Spectrogrammes (a$\mathbf{c}, \mathbf{g}-\mathbf{r})$ and frequency spectra $(\mathbf{d}-\mathbf{f}, \mathbf{j}-\mathbf{r})$ displaying coherence $(\mathbf{a}-\mathbf{f})$, wPLI $(\mathbf{g}-\mathbf{I})$, PLV $(\mathbf{m}-\mathbf{o})$ and PPC $(\mathbf{p}-\mathbf{r})$ along the PFC-dHC $(\mathbf{a}, \mathbf{d}, \mathbf{g}, \mathbf{j}, \mathbf{m}, \mathbf{p})$, PFC$\mathrm{VHC}(\mathbf{b}, \mathbf{e}, \mathbf{h}, \mathbf{k}, \mathbf{n}, \mathbf{q})$ and $\mathrm{VHC}-\mathrm{dHC}(\mathbf{c}, \mathbf{f}, \mathbf{i}, \mathbf{l}, \mathbf{o}, \mathbf{r})$ connections. Dotted red lines in spectra indicate boundaries of the analysed frequency bands named by the greek letters at the top. Stars indicate significant differences between genotypes ( $t$ test) in mean (black) or peak (grey) synchrony metrics. Lines display mean \pm SEM. ${ }^{*} p<0.05 ;{ }^{* *} p<0.01 ;{ }^{* * *} p \leq 0.001$

In reverse, in the gamma range, PFC led both hippocampal regions exclusively in the knockouts (Fig. 3j), which is not consistent with GC, but-at least for the PFCvHC connection-with PDC. Lastly, we examined the coherence phase angle. This showed a characteristic $90^{\circ}$ shift between the theta, beta and gamma oscillations of the PFC vis-à-vis the dHC, particularly in wild-type mice. In contrast to the other directional metrics, significant genotype-related differences were only seen in the gamma range, and they were prominent in the two $\mathrm{HC}$ PFC connections (Fig. 3k).

Finally, dHC- and vHC-gamma oscillations were coupled stronger to theta oscillations in PFC and the mutually coupled part of the hippocampus in knockouts (gamma-theta cross-regional PAC; Fig. 3l). However, PFC-gamma to hippocampal theta coupling was even reduced in knockouts (Fig. 3l) which contrasts sharply with the results from all other measures.

In summary, while the identification of genotyperelated differences in coupling was similar between some measures (especially coherence, PLV, PPC and GC), there was also a considerable lack of redundancy across the different measures of interregional connectivity (see overview in Table 2).

\section{Differences in detecting increases of inter-regional coupling over time in Gria1-knockouts across measures}

As a second indicator for redundancy between connectivity measures, we investigated the potential physiological correlates of the characteristic divergence of exploratory drive between the two genotypes over time (Fig. 1b, c). This divergence is likely induced by a failure of spatial short-term habituation in Gria1-knockout mice resulting in increasing exploration-as opposed to the decreasing activity seen in controls $[48,49]$. To allow for an efficient analysis, we captured the change of a given parameter over time in a single number, namely the slope of the linear interpolation across the time series over the 10-min test. We previously found that both local theta power in the $\mathrm{dHC}$ and also dHC-PFC theta coherence displayed a characteristic divergence between the groups that mirrored exploratory behaviour [48]. In this novel dataset and analysis, this pattern emerged much more broadly, namely across multiple power and coherence measures in all three connections (compare Fig. 1b, c with Fig. 4a-d). This included local PFC power in all analysed frequency bands and gamma and (at trend-level) theta peak power in the hippocampal regions (Fig. 4a, b). For coherence, the KO-related increase in slopes was limited to the delta and theta range and was apparent in the hippocampal-prefrontal connections (confirming our earlier results) and marginally for intra-hippocampal coupling (Fig. 4c, d). In the beta and gamma range, either no group difference occurred orfor PFC-dHC beta coherence-it was even inversed with a higher slope in wild-type mice. Stunningly, this pattern was not reproduced by the wPLI analysis (Fig. 4e, f) even in the one case where the coupling slope was increased in knockouts in both metrics (PFC-vHC, theta), the metrics differed in the respect that, in wild-type controls, theta-wPLI remained constant, while theta coherence decreased over time.

$G C$ remained largely constant or slightly decreased over time in wild-type mice, irrespective of connection or frequency band (Fig. 4g-i). In Grial $^{-1-}$ mice, in contrast, GC increased over time in the delta and theta range in most connections leading to genotype-related differences in the $\mathrm{vHC} \rightarrow \operatorname{PFC}(\delta, \theta), \quad \mathrm{vHC} \rightarrow \mathrm{dHC}(\theta), \mathrm{dHC} \rightarrow \mathrm{vHC}(\delta, \theta)$, $\mathrm{PFC} \rightarrow \mathrm{vHC}(\delta, \gamma)$ and $\mathrm{PFC} \rightarrow \mathrm{dHC}(\delta, \theta)$ projections. Thus, except for an isolated match in the vHC $\rightarrow$ PFC theta connectivity, the GC metric did not align with the wPLI-based slope assessment but provided a near-perfect match to the coherence slope pattern (Table 2). The latter observation even extends to the one instance of PFC-dHC beta coupling where the slope is higher in wild-type than in $\mathrm{KO}$ mice (Fig. 4g-i). The slope of the gamma-theta PAC also showed the expected divergence between genotypes in coupling strength along vHC connections, but not in the PFC-dHC connections (Fig. 4j). This pattern matched neither with coherence and GC (as they detected temporal changes in the PFC-dHC connection) nor with wPLI (which detected no changes in the vHC-dHC connection). Likewise, crosscorrelational lags did not change in any pattern that resembled the other measures (Fig. 4k). The slopes of MUArelated metrics were not determined because SPC analysis requires a considerable and equal number of spikes (not suitable for short intervals), and PDC and other lag metrics were not further regarded given that they already differed from the other metrics in the first comparison (Fig. 3).

\section{Lack of redundancy between most coupling measures revealed by bivariate correlation analysis}

Given that the above analysis of comparing genotyperelated differences across measures ultimately allows 


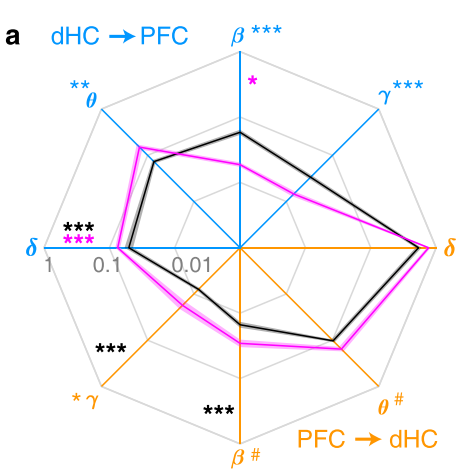

d

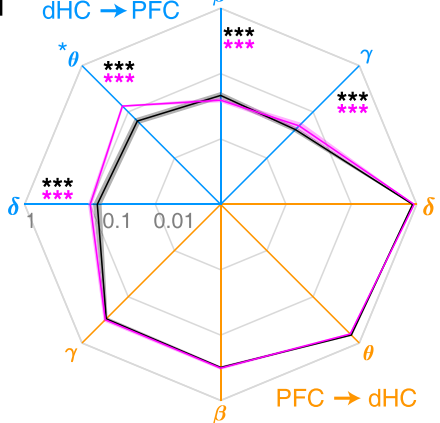

b

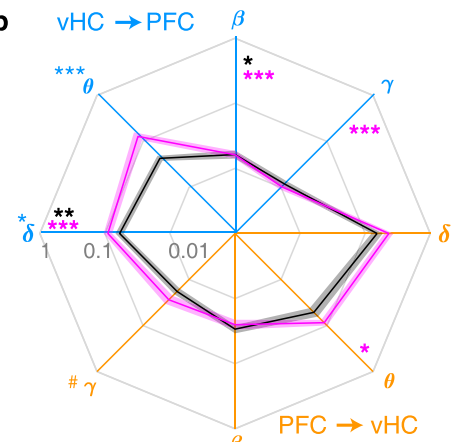

e

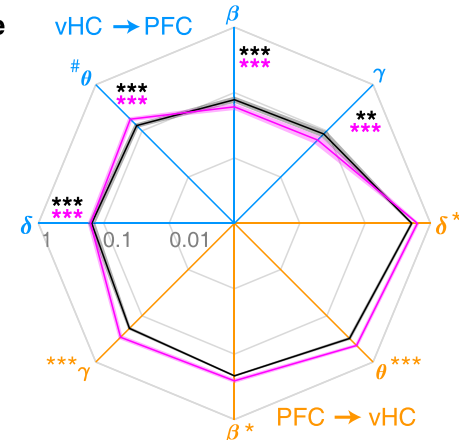

c $\quad \mathrm{vHC} \rightarrow \mathrm{dHC} \quad \beta \quad$ - $\quad$ KO

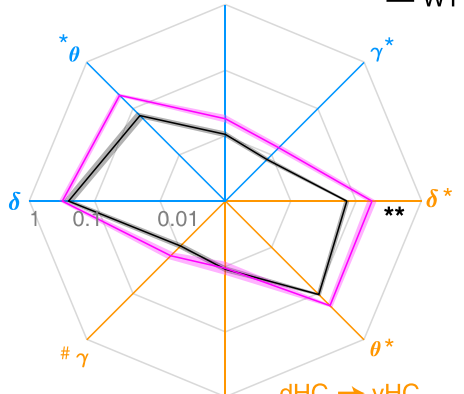

$\mathrm{dHC} \rightarrow \mathrm{vHC}$
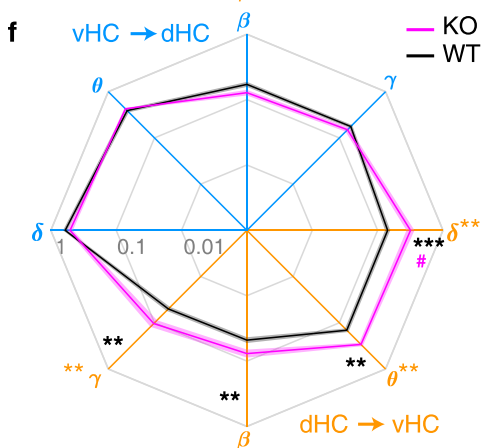

g Spike- $\theta$-phase $\mathbf{i}$ Lags of spike- $\theta$-phase j Lags of cross-amplitude coupling

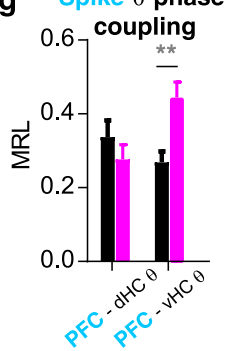

h
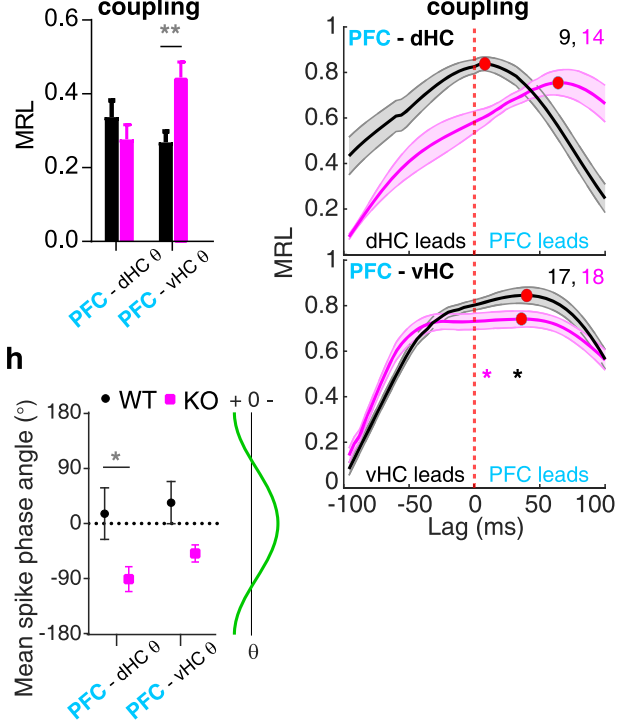

j Lags of cross-amplitude coupling -WT - KO ${ }^{1}$ PFC-dHC $: * * * \quad \theta[$ PFC-dHC $\quad \beta[$ PFC-dHC $\quad * \quad \gamma$

k $-\mathrm{WT}-\mathrm{KO}$

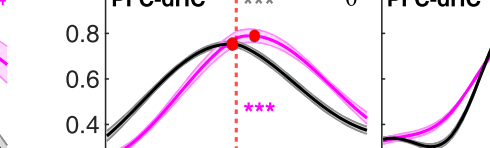

0.2

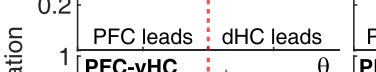

PFC leads $\mathrm{dHCleads}$ PFC leads $\mathrm{dHC}$ leads $\begin{array}{l:l}\text { PFC-vHC } & \beta\end{array}$ क⿺辶. 0.8
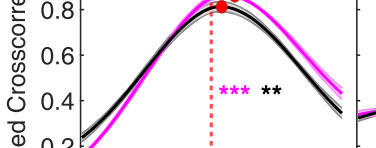

․․ㄹ

PFC leads vHC leads PFC leads vHCleads PFC

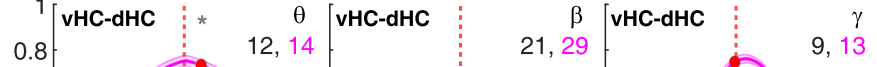

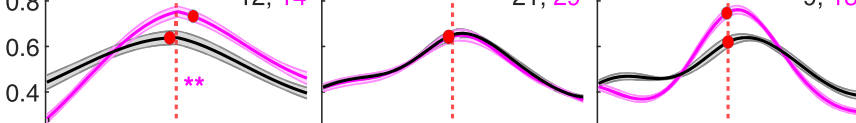

0.2 .

0 VHC leads $\mathrm{dHC}$ leads $\mathrm{VHC}$ leads $\mathrm{dHC}$ leads $\mathrm{vHC}$ leads $\mathrm{dHC}$ leads

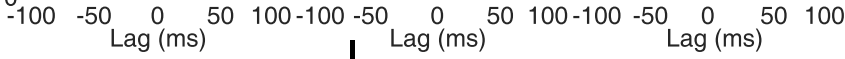

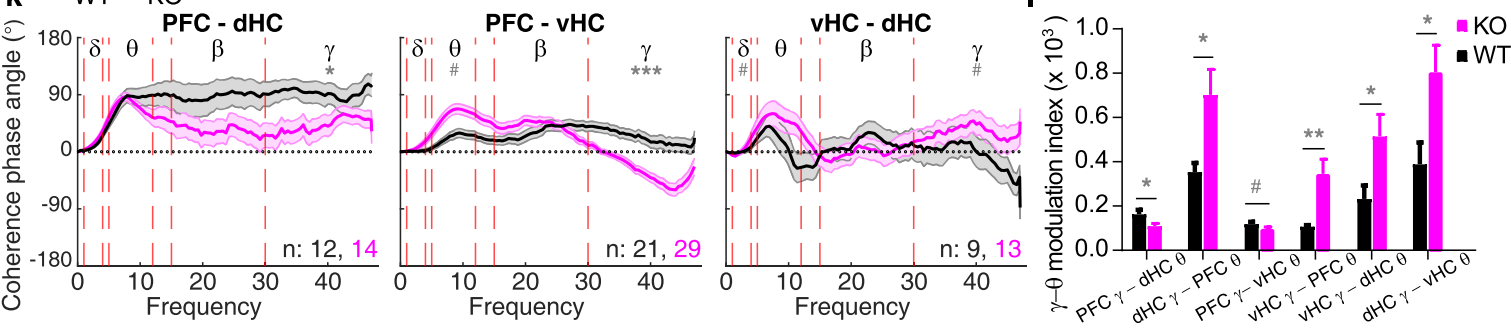

Fig. 3. (See legend on next page.) 
(See figure on previous page.)

Fig. 3. Directional metrics of inter-regional coupling in $\mathrm{Gria}^{-/-}$and wild-type controls across 10 min novelty-induced activity. a-c Parametric GC on $\log _{10}$ scale in the frequency bands indicated by greek letters and along the directional connections identified by the colour (blue: $\mathrm{dHC} \rightarrow \mathrm{PFC}$ $(\mathbf{a}), \mathrm{vHC} \rightarrow \mathrm{PFC}(\mathbf{b}), \mathrm{vHC} \rightarrow \mathrm{dHC}(\mathbf{c})$; orange: reverse of the before). Statistical indicators in the same colour identify a difference between genotypes (Sidak); statistical indictors in black (WT) or purple (KO) refer to a significant difference between the GC values of the two opposing directions within the colour-coded genotype whereby the location of the indicator identifies the direction with smaller average GC. $\mathbf{d}$-f The same display as a-c but for PDC. g Mean resultant vector length (MRL) as an indicator of SPC of prefrontal spikes to hippocampal theta. $\mathbf{h}$ Average theta phase angle of the mean resultant vector from SPC analysis. The theta phase corresponding to the degree value is shown on the right (horizontal axis illustrates voltage of LFP). i MRL as a function of lag between prefrontal MUA and hippocampal LFP. Some data was excluded based on lag amplitudes above 100 ms; contributing $N$ numbers are stated; statistics identical to $\mathbf{g}$. $\mathbf{j}$ Cross-correlation functions of instantaneous amplitude curves in the connections and frequency bands named at the top of each sub-panel with peak values indicated by a red dot. Statistical indictors in black (WT) or purple (KO) refer to a significant difference of the lag (temporal shift) from 0 ms (Wilcoxon's signed rank test). $\mathbf{k}$ Spectra of coherence phase angle along the named connection. Dotted red lines and greek letters indicate analysed frequency bands. I Theta-gamma crossregional PAC for the named directional connections. Solid lines display mean, and shaded area SEM throughout; bars display mean \pm SEM throughout. Grey stars in $\mathbf{g - I}$ indicate genotype differences ( $t$ test in $\mathbf{g}, \mathbf{i}, \mathbf{j}$ and $\mathbf{I}$; Watson-Williams test in $\mathbf{h}$ and $\mathbf{k}$ ). ${ }^{\#} p<0.1 ;{ }^{*} p<0.05$; ${ }^{* *} p<0.01 ;{ }^{* * *} p \leq 0.001$

Table 2 Pairwise comparison between wild-type and Gria1-knockouts

\begin{tabular}{|c|c|c|c|c|c|c|c|c|c|c|c|c|c|}
\hline \multirow[t]{2}{*}{ KO vs. WT } & & \multicolumn{3}{|l|}{ Delta } & \multicolumn{3}{|c|}{ Theta } & \multicolumn{3}{|l|}{ Beta } & \multicolumn{3}{|c|}{ Gamma } \\
\hline & & $\begin{array}{l}\text { PFC- } \\
\text { dHC }\end{array}$ & $\begin{array}{l}\text { PFC- } \\
\text { vHC }\end{array}$ & $\begin{array}{l}\text { vHC- } \\
\text { dHC }\end{array}$ & $\begin{array}{l}\text { PFC- } \\
\text { dHC }\end{array}$ & $\begin{array}{l}\text { PFC- } \\
\text { vHC }\end{array}$ & $\begin{array}{l}\text { vHC- } \\
\text { dHC }\end{array}$ & $\begin{array}{l}\text { PFC- } \\
\text { dHC }\end{array}$ & $\begin{array}{l}\text { PFC- } \\
\text { vHC }\end{array}$ & $\begin{array}{l}\text { vHC- } \\
\text { dHC }\end{array}$ & $\begin{array}{l}\text { PFC- } \\
\text { dHC }\end{array}$ & $\begin{array}{l}\text { PFC- } \\
\text { vHC }\end{array}$ & $\begin{array}{l}\text { vHC- } \\
\text { dHC }\end{array}$ \\
\hline \multirow{15}{*}{$\begin{array}{l}\text { Average } \\
\text { metric }\end{array}$} & Coherence & & & & $* * *$ & $* * *$ & $* * *$ & & & & * & & \\
\hline & WPLI & $* *$ & & * & $*$ & $* * *$ & $* *$ & $* *$ & $\#$ & & * & * & $\#$ \\
\hline & PLV & & & & $* * *$ & $* * *$ & $* * *$ & * & & & $* *$ & & \\
\hline & PPC & & & & $* * *$ & $* * *$ & $* * *$ & * & & & $* *$ & & \\
\hline & $\mathrm{GC} \rightarrow$ & & & & $\#$ & & * & $\#$ & & & * & $\#$ & * \\
\hline & $\mathrm{GC} \leftarrow$ & & * & * & $* *$ & $* * *$ & * & $* * *$ & & & $* * *$ & & $\#$ \\
\hline & $\mathrm{PDC} \rightarrow$ & & * & & & $* * *$ & & & * & & & $* * *$ & \\
\hline & $\mathrm{PDC} \leftarrow$ & & & $* *$ & * & $\#$ & $* *$ & & & & & & $* *$ \\
\hline & $M R L \leftarrow$ & & & & & $* *$ & & & & & & & \\
\hline & $\mathrm{Ml} / \mathrm{PAC} \rightarrow$ & & & & * & $* *$ & * & & & & & & \\
\hline & $\mathrm{Ml} / \mathrm{PAC} \leftarrow$ & & & & * & \# & * & & & & & & \\
\hline & $\begin{array}{l}\text { Coher. } \\
\text { phase }\end{array}$ & & & $\#$ & & $\#$ & & & & & * & $* * *$ & $\#$ \\
\hline & CC & & & & $* * *$ & * & * & & & & * & $* * *$ & \\
\hline & $\begin{array}{l}\text { MRL-phase } \\
\leftarrow\end{array}$ & & & & * & & & & & & & & \\
\hline & MRL-lag $\leftarrow$ & & & & & & & & & & & & \\
\hline \multirow[t]{7}{*}{ Slope metric } & Coherence & $* * *$ & $* *$ & * & $* *$ & $* * *$ & $\#$ & $* *$ & & & & $\#$ & \\
\hline & WPLI & $\#$ & & & & $* *$ & & & & & & & \\
\hline & $\mathrm{GC} \rightarrow$ & $* *$ & * & & $* *$ & & * & * & & & & $* *$ & \\
\hline & $\mathrm{GC} \leftarrow$ & & $* *$ & $* *$ & & $* * *$ & * & & & & & & * \\
\hline & $\mathrm{MI} / \mathrm{PAC} \rightarrow$ & & & & & $* *$ & & & & & & & \\
\hline & $\mathrm{Ml} / \mathrm{PAC} \leftarrow$ & & & & & * & * & & & & & & \\
\hline & $\mathrm{CC}$ & & & & * & & & $\#$ & & & & & * \\
\hline
\end{tabular}

Overview over genotype-related statistical comparisons of the data displayed in Figs. 2, 3 (average metric) and 4 (slope metric). GC and PDC results are derived from the Sidak post hoc test after repeated-measures ANOVA across both directions of a connection and genotypes; MRL-phase and coherence-phase angle are compared with the Watson-Williams test [20]; all other $P$ values are derived from independent-sample $t$ tests. For LFP-based measures (coherence, wPLI, PLV, PPC, GC, PDC), the $P$ values in the theta range refer to the peak theta (not mean theta). Arrows in directional measures indicate direction of coupling: $\rightarrow$ direction labelled in column name (e.g. PFC $\rightarrow \mathrm{dHC}$ in the PFC-dHC column); $\leftarrow$ opposite direction. For Ml and MRL measures, the region named first corresponds to the region that contributes the theta oscillation to the analysis. $p \geq 0.1 ;{ }^{*} p<0.1 ;{ }^{*} p<0.05,{ }^{* *} p<0.01,{ }^{* * *} p \leq 0.001$ 


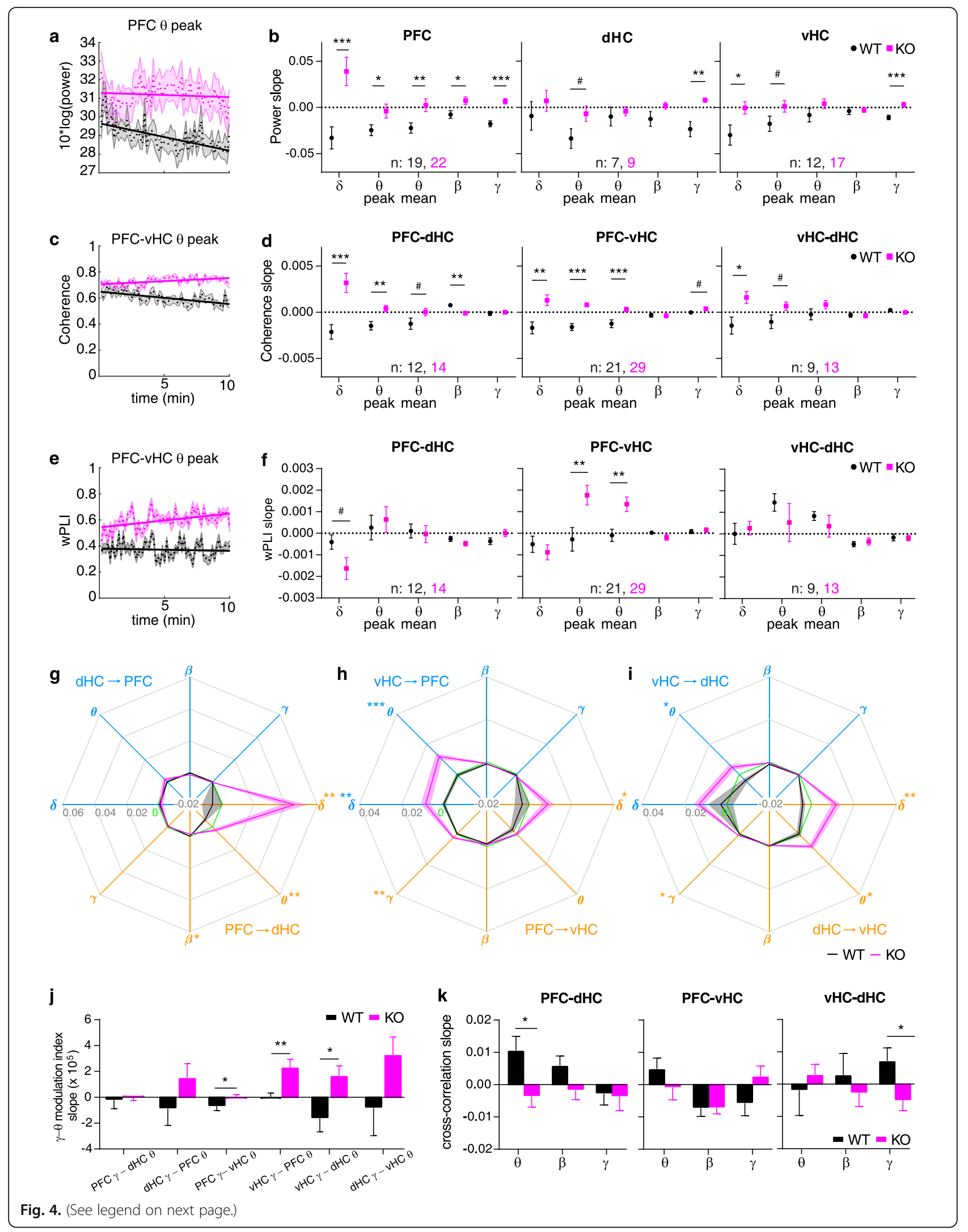


(See figure on previous page.)

Fig. 4. Changes of power and coupling strength over time during the 10 min open-field test. a, $\mathbf{c}$, e Examples of individual measures of power (a), coherence (c) and WPLI (e) as they behave as population average over the $10 \mathrm{~min}$ of novelty-induced activity in the open field (dashed line, mean; shaded area, SEM) with linear interpolation between time points overlaid (solid line) to determine the slope as indicator of temporal changes. $\mathbf{b}, \mathbf{d}$, f Average slope (temporal change) of power (b), coherence (d) and wPLI (f) in the indicated regions or connections (top of subpanel) and frequency bands ( $x$-axis). $\mathbf{g}, \mathbf{h}$, i Slope of GC in the frequency bands indicated by greek letters and along the directional connections identified by the colour (blue: $\mathrm{dHC} \rightarrow \mathrm{PFC}(\mathbf{g}), \mathrm{vHC} \rightarrow \mathrm{PFC}(\mathbf{h}), \mathrm{vHC} \rightarrow \mathrm{dHC}(\mathbf{i})$; orange: reverse of the before). Statistical indicators in the same colour identify a difference between genotypes ( $t$ test). $\mathbf{j}$ Slope of theta-gamma PAC in the stated directional connections. $\mathbf{k}$ Slope of cross-correlation lags indicating putative changes of temporal shifts of the oscillations in the named frequency bands. Black stars indicate significant differences between genotypes ( $t$ test), and error bars or shaded regions indicate SEM throughout. ${ }^{\#} p<0.1 ;{ }^{*} p<0.05 ;{ }^{* *} p<0.01 ;{ }^{* * *} p \leq 0.001$

only a qualitative judgement about the epistemological redundancy of interregional coupling metrics, we supplemented our analysis by a more quantitative analysis in form of bivariate Spearman correlations between pairs of parameters and within genotypes and connections using the average value for each parameter in each electrode pair as a dependent variable. We included all metrics analysed in Figs. 2 and 3 and also partial directed coherence (PDC) and non-parametric Granger causality (npGC). This revealed multiple levels of complexity when analysing the relation between the metrics. On the one hand, at the level of isolated observations, the correlations supported the commonalities between measures already seen with the two prior analyses. For example, PFC-dHC theta coherence correlated strongly with $\mathrm{dHC} \rightarrow$ PFC theta-GC in wild-types (Fig. 5a). However, this correlation did neither exist in the knockouts in the same connection (Fig. 5a) nor in the same genotype but the PFC- $v H C$ connection (Fig. 5b). Indeed, PFC-vHC theta coherence did correlate highly with GC in the opposite, i.e. $\mathrm{PFC} \rightarrow \mathrm{vHC}$, direction but not in the $\mathrm{vHC} \rightarrow$ PFC direction-and it did so across all frequency bands (Fig. 5b, see Additional file 1: Table S1 and S2 for the full correlation tables in wild-type mice including all metrics and four frequency bands), which was not the case in the other two connections (Figs. 5a and 6a). In general, when carefully examining each pair of metrics, it became apparent that a correlation seen in one genotype and connection would rarely reappear in another one (Fig. 5a, b, 6a).

In order to evaluate this systematically, we calculated the average correlation coefficient for each pair across the three connections and indicated its significance only if it was given in all of them (Fig. 6b). Reassuringly, the three pairs of mathematically closely related metrics showed consistent correlations in each connection and frequency band: PPC and PLV, parametric and nonparametric GC, and PDC and DTF. Beyond that, however, there was not a single pair of distinct metrics that achieved a significant correlation in all three connections in wild-type mice in the theta band, and only two (coherence correlating with PPC and PLV) in the gamma band (Fig. 6b, Additional file 1: Table S4). In Gria1knockouts, the picture was similar, except that, here, coherence correlated significantly with PLV and PPC in both the theta and the gamma bands, and additionally, gamma wPLI correlated with coherence, PPC and PLV, across connections. The latter result contrasts sharply with the absence of such wPLI correlations in wild-type mice, illustrating that some observed correlations may depend on the genotype and are hence not reflecting a priori redundancies.

We further examined the correlations that were not significant in all three connections, but yet achieved a high correlation coefficient on average. In the theta range, coherence also correlated strongly with $\mathrm{PPC}$ and PLV (average rho $\geq 0.8$ ) -in alignment with our first analysis (Figs. 2 and 3), the correlation result in knockouts, and the gamma band in both genotypes (Fig. 6b) and with coherence phase angle (average rho $>0.7$ ); further correlations yielded a medium (0.6-0.7) average rho: (a) coherence phase angle with PPC, PLV, PDC, DTF, GC and npGC and (b) PPC/PLV with wPLI, PDC and DTF. In the gamma range, coherence phase angle also showed the largest number of medium average correlations with other measures, namely wPLI (average rho $=0.77)$ and with coherence magnitude, PPC, PLV, PDC, DTF, GC and npGC (average rho 0.6-0.7); the only remaining medium average correlations $(0.6-0.7)$ in the gamma range were wPLI with PDC and DTF (Fig. 6b, Additional file 1: Table S4). Also in knockouts, the coherence phase angle showed average medium correlations with most other LFP-based metrics in the theta and gamma range (Fig. 6b). It should be noted that this combined analysis may overlook correlations with directional metrics in case they occur in only one direction. For example, theta GC (and npGC) did actually correlate with theta PDC (and DTF) in each of the three connections but only in one direction each: $\mathrm{PFC} \rightarrow \mathrm{vHC}, \mathrm{dHC} \rightarrow \mathrm{PFC}$ and $\mathrm{vHC} \rightarrow \mathrm{dHC}$ which is difficult to interpret given that we always recorded significant GC and PDC in both directions. Results from the SPC (MRL), PAC and amplitude cross-correlation (lag) analyses did not correlate with any other measure consistently in any genotype. This synopsis largely aligns with the redundancy patterns seen with the two former analyses (Table 2). 


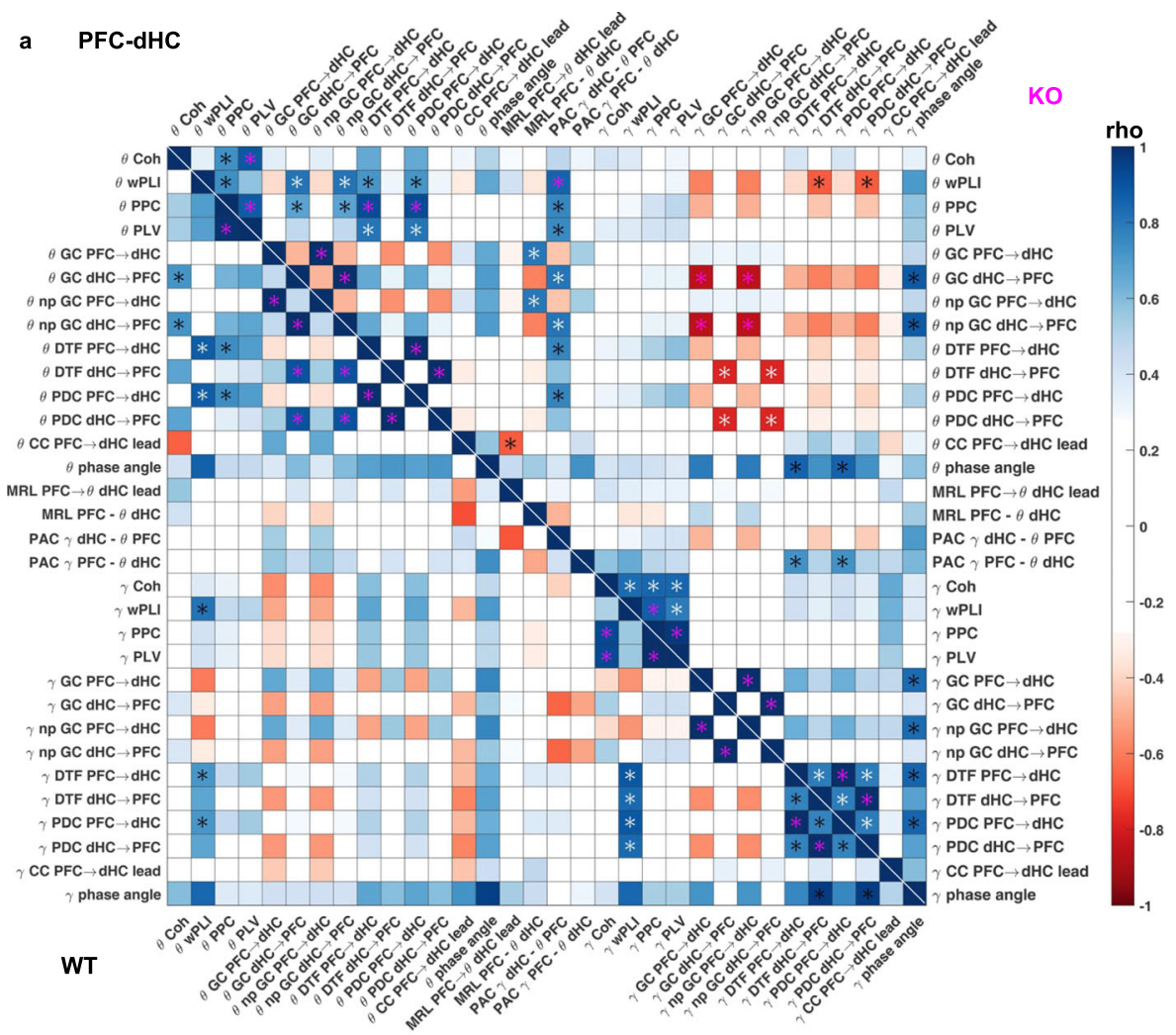

b PFC-vHC

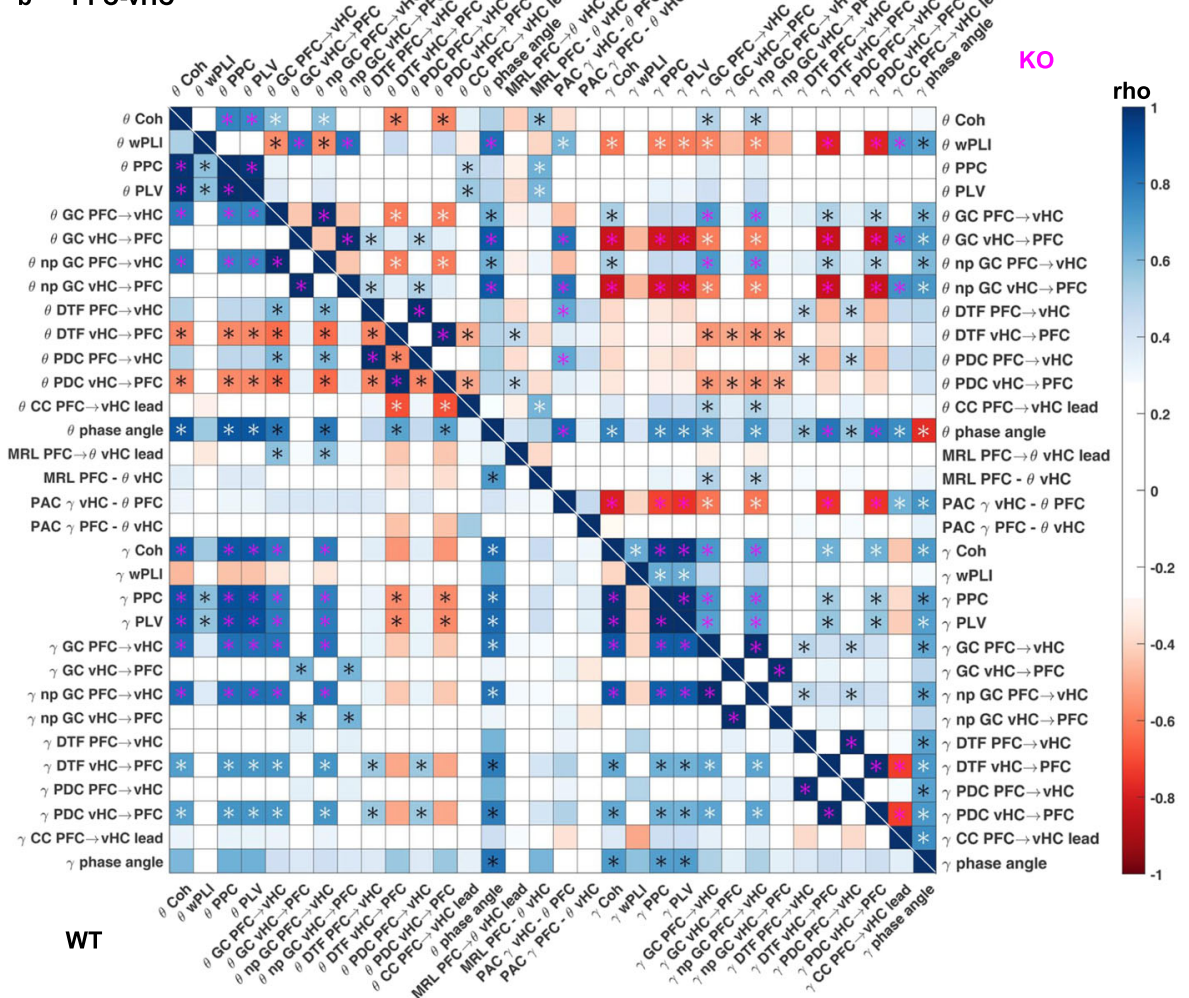

Fig. 5. (See legend on next page.) 
(See figure on previous page.)

Fig. 5. Correlations between individual measures of hippocampal-prefrontal connectivity. a, b Spearman's coefficient (rho, colour of squares) and significance (star within squares) of bivariate correlations between individual measures of connectivity in the PFC-dHC (a) and PFC-vHC (b) connections within KO (top-right) and WT (bottom-left) mice. White stars, $p<0.01$; purple stars, $p<0.001$. Theta and gamma metrics are spatially separated, and delta and beta metrics are omitted (see Additional file 1: Table S1 and S2 for pairwise correlations of all metrics analysed in this figure)

\section{Sensitivity of measures to reference location}

The choice of placement site for the reference electrode varies considerably between studies, and both the referencing to the ground screw above the cerebellum (as done for all above analyses) and to the anterior part of the frontal cortex are widely used. In order to investigate the effect of this difference, we recorded a separate reference signal from a frontal reference screw [2, 45] and used it to digitally re-reference all recorded data by subtracting this signal from the recorded LFP traces before re-calculating the local power, coherence, wPLI and GC.

Using repeated-measure ANOVAs with the withinsubject factor of re-referencing and the between-subject factor of genotype, we found that the location of the reference has quite a substantial influence on the results. There were significant effects of re-referencing on delta power, coherence and GC in all brain regions (except for the MD) and connections, while the effect on wPLI was comparatively minor (but note that delta wPLI is generally very low and entirely different from delta-coherence and GC; Fig. $7 \mathrm{a}-\mathrm{m}$ ). In the theta range, in contrast, rereferencing affected power only in the $\mathrm{dHC}$ but strongly impacted coherence, wPLI and GC alike along both hippocampal-prefrontal connections-not only in terms of significant effects of re-referencing, but also in terms of genotype-reference interactions, which indicate that the prior conclusions on theta range connectivity are partly dependent on the position of the reference. In the GC measure, interactions were apparent in the $\mathrm{d} /$ $\mathrm{vHC} \rightarrow$ PFC direction but not in the reverse (Fig. 7k, l). Nevertheless, there were also significant effects of genotype in those connections and measures, suggesting that the fundamental observation of elevated hippocampalprefrontal theta connectivity in knockouts still holds, especially for the PFC-dHC connection and the GC measure in general (Fig. 7e, f, h, i, k, l). Intra-hippocampal theta connectivity was not much affected by the reference placement, irrespective of measure (Fig. $7 g, j, \mathrm{~m}$ ).

In the higher frequency ranges, the effects were more mixed. Beta power in the $\mathrm{dHC}$ and coherence-but only partly wPLI and GC-along its connections were affected by reference placement. In the gamma range, rereferencing impacted power in the PFC and $\mathrm{dHC}$, wPLI in the PFC-d/vHC connections and coherence along all three connections (Fig. $7 \mathrm{a}-\mathrm{j}$ ). In fact, the formerly observed lower PFC-dHC gamma coherence and wPLI in knockouts (Fig. 2d, j) were dependent on the reference placement for detection (interaction effect only for coherence and wPLI, Fig. 7e, h). A similar observation holds for the PFC-vHC gamma connectivity which was increased in KOs in the wPLI, but not the coherence measure (Fig. 2e, k). Here again, an interaction indicated that the absence or presence of this difference in the coherence measure depends on the reference location (Fig. 7f), while an effect of genotype is maintained when using wPLI even though an interaction is found in addition (Fig. 7i). The impact of referencing on gamma$\mathrm{GC}$, in contrast, was limited to the $\mathrm{dHC} \rightarrow \mathrm{PFC}$ projection (Fig. $7 \mathrm{k}-\mathrm{m}$ ).

In summary, a frontal reference screw-as often used when studying prefrontal-hippocampal connectivity [2, 45] - may considerably alter the results obtained for LFP-based measurements of connectivity between the PFC and the hippocampus. Somewhat surprisingly, the wPLI measure does not eliminate this contingency but only reduces it, especially in the beta-gamma range. Referencing effects on GC are particularly visible in the low (delta/theta) frequency range and (as interactions) in the direction from the hippocampus to PFC.

\section{Discussion}

We here examined the level of redundancy and experimental contingencies of the most widely applied measures of interregional directed and non-directed neuronal connectivity that are obtainable with chronically implanted field electrodes in awake rodents. This analysis revealed a surprisingly large absence of redundancies between such metrics and a worrying contingency with respect to the location of the reference electrode. Both findings suggest that the implicitly held belief that experimental results obtained with one metric of connectivity and one configuration for referencing would allow general conclusions about aberrations in inter-regional functional connectivity is problematic. Intriguingly, a similar conclusion has been reached by a recent study on connectivity measures applied on human EEG data [50].

While this finding was somewhat expected a priori when regarding metrics of distinct conceptual foundation-e.g. non-directional synchrony vs. measures of causation-the lack of similarity even within the same analytical category is unreckoned. From a conceptual perspective, the result reveal the absence of a concrete empirical counterpart of the rather interchangeably used 

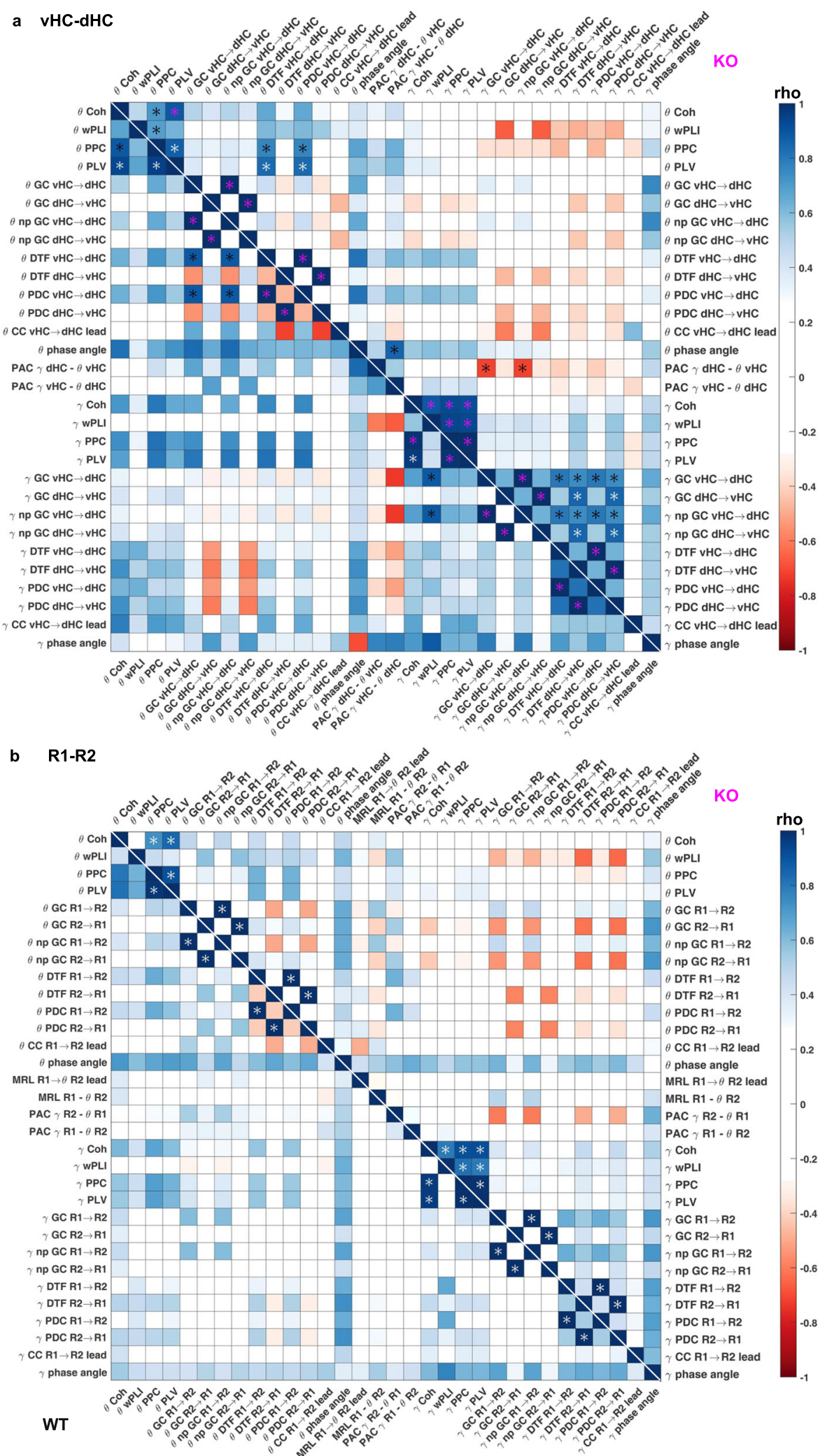

Fig. 6. (See legend on next page.) 
(See figure on previous page.)

Fig. 6. Correlations between individual measures of intra-hippocampal and overall connectivity. a Spearman's coefficient (rho, colour of squares) and significance (star within squares) of bivariate correlations between individual measures of connectivity in the vHC-dHC connection within KO (top-right) and WT (bottom-left) mice. $\mathbf{b}$ The same display as in $\mathbf{a}$ but indicating the average correlation coefficient across the three connections (Figs. $5 a, b$ and $6 a$ ) by the colour of a square and significance only if a significant correlation existed in every one of the three connections. White stars, $p<0.01$; purple stars, $p<0.001$. Theta and gamma metrics are spatially separated, and delta and beta metrics are omitted (see

Additional file 1: Table S3 and S4 for pairwise correlations of all metrics analysed in this figure)

terms of inter-regional communication, coupling, information transfer or functional connectivity. Given these contingencies of a result obtained with any single metric, it is difficult to equate it with the too generic notion of neuronal communication.

A particular analytical problem appears to be the lack of benchmarking of the sensitivity, specificity and robustness of the individual measures against a ground truth of actual physiological trans-synaptic activity along anatomically verified connections. Notably, we here, like previous studies, found evidence for significant causal influence not only along the direct anatomical projections $-\mathrm{dHC} \rightarrow \mathrm{vHC} \quad[51,52], \quad \mathrm{vHC} \rightarrow \mathrm{dHC} \quad[52]$ and $\mathrm{vHC} \rightarrow$ PFC [52-55]-but also along the PFC-dHC connection that is mediated only indirectly via the nucleus reuniens [56, 57], and even in the direction for which no obvious anatomical correlate has been described yet to our knowledge (PFC $\rightarrow \mathrm{vHC}$ [52]), which complicates the validation and interpretation of the functional connectivity measurements.

In the absence of such benchmarking and while facing considerable logistical limits in applying multiple referencing and metrics for every experiment, our analysis at least qualitatively implies some guidelines to choose the set of coupling metrics suited for a rather comprehensive, yet non-redundant analysis of inter-regional communication.

Firstly, we demonstrate that some mathematically related measures do actually show a pairwise redundancy and hence do not need to be included into the same analysis, namely PPC and PLV [30], parametric and nonparametric GC (allowing for considerably faster computation by using the non-parametric approach [15]) and PDC and DTF $[16,18]$.

Secondly, beyond these reliable redundancies, we found further partial redundancies across connections, genotypes and frequencies helping to narrow the list of metrics to include in an analysis further. Most importantly, PPC and PLV also showed considerable overlap with both the magnitude and (to a lesser degree) the phase angle of coherence, and medium average correlations with wPLI, PDC and DTF. In addition, coherence phase angle correlated broadly at a medium average level with coherence amplitude, PDC, DTF, GC and npGC in addition to PLV and PPC. For practical purposes, this suggests that an assessment of two metrics-PPC and coherence phase angle-would be a useful first-pass approach to survey LFP data for possible aberrations in functional connectivity, which can then be followed up with mutually non-redundant directional metrics.

Thirdly, while in such further analysis, GC and PDC (or DTF) may seem particularly attractive metrics given that they deliver a more fine-grained picture of coupling in distinct directions and may be interpreted as indicators of causal influence between two brain regions, it is important to note that they do not yield similar results even though they are sometimes (erroneously [58]) equated. Despite some correlations between PDC (and DTF) with PLV, PPC and coherence phase angle in the correlation analysis (Figs. 5 and 6), there were actually considerable and irresolvable discrepancies between these measures in the genotype comparison (compare Fig. 2 with Fig. 3d-f); for example, genotype-related differences in PFC-dHC gamma-range coupling seen across all measures of synchrony and coherence phase angle were not detected by $\mathrm{PDC}$, while the reverse was true for the $\mathrm{vHC}-\mathrm{dHC}$ connection. $\mathrm{GC}$, in contrast, did mostly reflect aberrations seen with the synchrony measures and could clarify their directional underpinning (Table 2). Therefore, PDC/DTF and GC may serve as complementary metrics rather than surrogates.

Fourth, spike-phase and phase-amplitude coupling cannot be expected to be equivalent to any of the other parameters and are therefore very useful to include to deliver a different perspective on functional connectivity. While this may have been expected given their distinct biological nature, the degree of absence of redundancy is nevertheless astonishing. It should be noted, however, that the presented SPC analysis using MUA [21] is likely far from optimal given that units cannot be chosen by the movement of the electrodes and not properly sorted. The recording of single-unit activity from moveable electrode bundles or arrays $[2,45]$ will certainly improve the assessment of SPC and its related directional measures.

Finally, for LFP-based measures, the reference electrode should be placed in a brain structure that is largely separate from the brain regions between which connectivity is studied. A frontal screw may easily obscure phenotypes in prefrontal connectivity as it may pick-up field potential signals from the PFC $[29,59]$.

\section{Conclusions}

In summary, our analysis calls for a more cautious interpretation of previous findings in the rodent literature on 


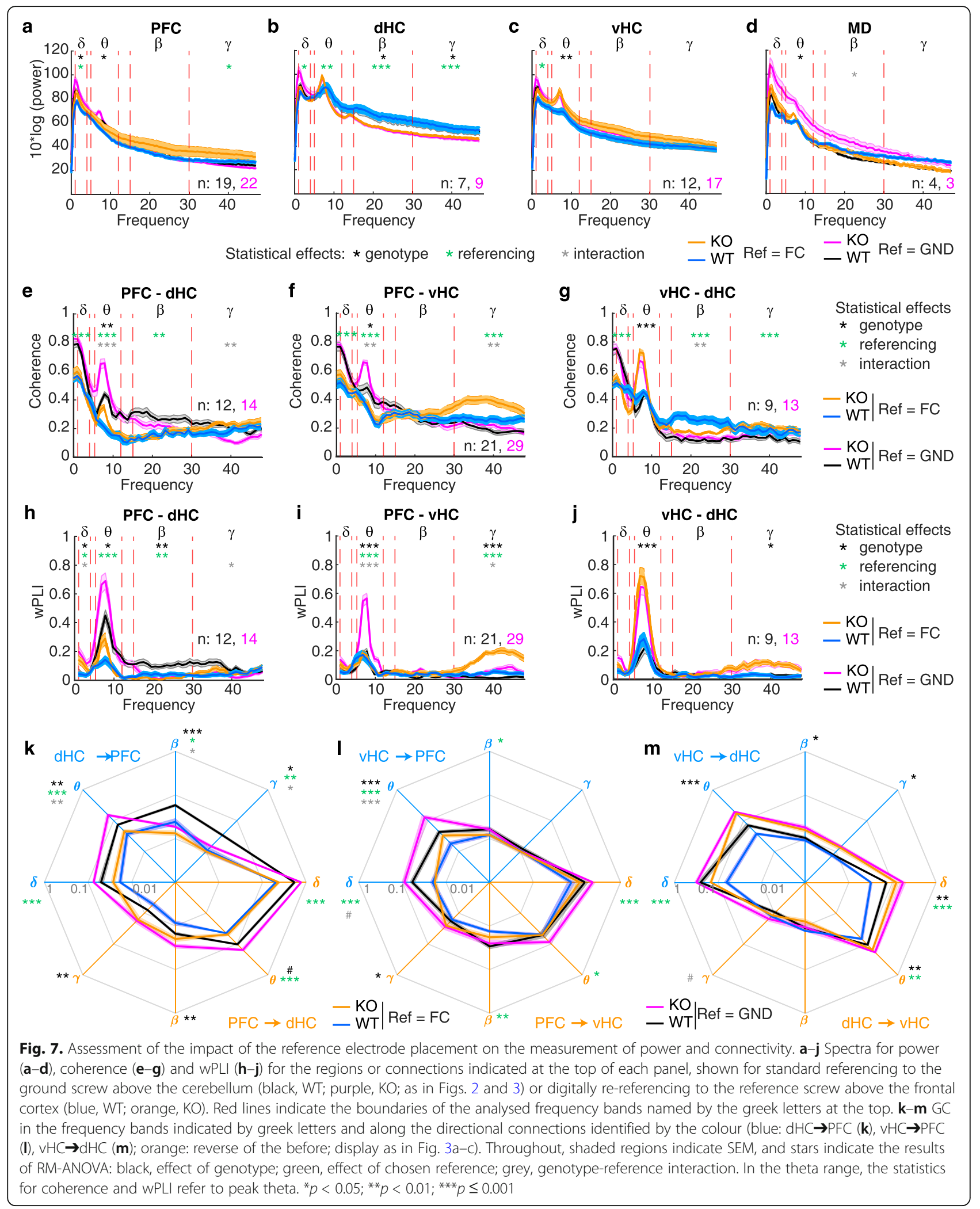


inter-regional coupling (especially when regarding negative results), the need for better benchmarking of individual measures and the necessity to report multiple measures of connectivity in future studies.

\section{Methods}

Animals

Male and female Gria1 knockout (Gria1 ${ }^{-/}$, Grial ${ }^{\text {tm1Rsp; }}$ MGI:2178057) [60] mice ( $N=15,9$ males) and wild-type littermate controls ( $N=12,8$ males) were bred from heterozygous parents. Animals were group-housed in type II long individually ventilated cages (Greenline, Tecniplast, G), enriched with sawdust, sizzle-nest ${ }^{\mathrm{tu}}$ and cardboard houses (Datesand, UK) and subjected to a 13-h light/11-h dark cycle. The mice were implanted with electrodes at ca. 9 months of age and were tested in the open-field test ca. 3-5 weeks later to allow recovery from surgery intermittently.

\section{Surgery}

Electrode implantation surgeries under general isoflurane anaesthesia and a broad peri-operative analgesic regime were conducted similarly as previously described for a similar dataset from a distinct cohort [48]. Briefly, single polyimide-insulated tungsten wires of $50 \mu \mathrm{m}$ diameter (WireTronic Inc., CA, USA) were implanted, with reference to the bregma (in $\mathrm{mm}$ ), into the PFC (AP + 1.8-1.9, ML 0.3-0.35; 1.8-1.9 below pia), MD (AP 1.2, ML 0.3, 2.7 below pia), dHC (AP - 1.9-2.0, ML 1.5, 1.4 below pia) and vHC (AP - 3.1-3.2, ML 2.9-3.0, 3.4 $\mathrm{mm}$ for single and $3.8-3.9 \mathrm{~mm}$ for dual electrodes below pia). In a majority of mice, dual electrodes were used for PFC and $\mathrm{vHC}$, whereby the second electrode was placed about $0.5 \mathrm{~mm}$ higher than the stated distance from pia. In later analysis, the data from each electrode was regarded as the unit of observation $(N)$, so that a single mouse could contribute up to an $N=4$ for vHC-PFC connections and up to an $N=2$ for dHC-vHC, PFCvHC, MD-PFC and MD-vHC connections. Both hemispheres were implanted at roughly equal proportion. Stainless steel screws $(1.2 \mathrm{~mm}$ diameter, Precision Technologies, UK) were implanted in the contralateral hemisphere ca. $1 \mathrm{~mm}$ from the midline above the cerebellum (AP - 5.5) for ground and above the anterior frontal cortex $(\mathrm{AP}+4.0)$ for additional reference, and were connected with a 120- $\mu$ m PTFE-insulated stainless steel wire (Advent Research Materials Ltd., UK; Fig. 1a). All electrode wires were connected to pins in a dual-row 6pin or 8-pin connector (Mill-Max, UK).

To later determine electrode placements post-mortem, electrolytic lesions were made after breathing ceased under terminal ketamine/medetomidine anaesthesia. Immediately afterwards, animals were transcardially perfused with PBS followed by $4 \%$ paraformaldehyde (PFA)/
PBS, and the brains were post-fixed for $24 \mathrm{~h}$ in PFA/ PBS. Coronal sections of $60 \mu \mathrm{m}$ were cut on a vibratome in PBS and then washed 3 times in PBS, stained with DAPI and mounted for inspection of lesion sites on an epifluorescence microscope (DM6, Leica).

\section{Novelty-induced locomotion and recording}

Animals were tethered to enable electrophysiology recordings and then placed into a novel environment consisting of a clear type III plastic cage (length $43 \mathrm{~cm}$, width $22 \mathrm{~cm}$, height $20 \mathrm{~cm}$; Tecniplast) containing clean sawdust. Animals were allowed to explore for $10 \mathrm{~min}$. The animals' location in the open field was videotracked with ANY-maze (Stoelting, UK), and the distance travelled was calculated in $20 \mathrm{sec}$ time bins. Prior to testing, a 32-channel RHD2132 headstage (Intan Technologies, CA, USA) was plugged into the implanted connector via a custom-built adaptor that interfaced a 36-pin Omnetics connector (A79022-001, MSA components, G) with another 6-pin or 8-pin Mill-Max connector. The headstage was wired to an Open-Ephys acquisition board (https://open-ephys.org, USA; obtained through the Open-EPhys store at Champalimaud, Portugal) via two light-weight flexible SPI-cables (Intan Technologies), daisy-chained through a customconnected miniature slip-ring (Adafruit, NY, USA). The adaptor was wired so that all signals were referenced to the ground signal obtained from above the contralateral cerebellum, while the signal from the additional frontal reference screw was recorded separately (for later offline re-referencing) like the LFP channels, i.e. also referenced to ground. Using the RHD2132 headstage, the OpenEphys acquisition board and the Open-EPhys acquisition software, data were amplified and digitized, sampled at $10 \mathrm{kHz}$ and digitally high-pass filtered at $0.1 \mathrm{~Hz}$ for the acquisition of raw data (for MUA and GC analysis) and simultaneously band-pass filtered at $0.1-250 \mathrm{~Hz}$ (for all remaining analysis of LFP signals).

\section{Data processing and analysis}

All signal analyses were done in MatLab (MathWorks). Data were exported to MatLab and, for all LFP analyses, down-sampled to $1 \mathrm{kHz}$ and analysed with customwritten scripts. To reduce low-frequency drift, signals were first detrended using the locdetrend function of the Chronux signal processing toolbox (http://chronux.org/) with $1 \mathrm{~s}$ of data and a sliding window of $0.5 \mathrm{~s}$.

\section{Spectral analysis}

Power and coherence spectra as well as the phase angles were calculated with Chronux routines implemented in the Chronux toolbox using the multi-taper method [61]. Power values were expressed as $10 * \log _{10}$ values for all analyses, and the range of frequencies was set from 0.1 
to $48 \mathrm{~Hz}$. A bandwidth of $0.2 \mathrm{~Hz}$ and a total of $220 \mathrm{ta}$ pers were used to calculate power and coherence over the course of the $10 \mathrm{~min}$ exploration time. To analyse the temporal development, power and coherence were also calculated in 10-s bins using a bandwidth of $1 \mathrm{~Hz}$ and 19 tapers.

\section{Weighted phase lag index}

To address the issue of volume conduction, we calculated the weighted Phase Lag Index (wPLI) [5] using the routines implemented in the FieldTrip toolbox [62]. The 10-min exploration time was divided into nonoverlapping 1-s bins and padded to the next power of two. The complex cross-spectrum was computed using a Hann taper with a spectral smoothing of $0.5 \mathrm{~Hz}$. For temporal analysis, wPLI was averaged for each minute of the 10-min period using the same spectral parameters.

\section{Phase-locking value and pairwise-phase consistency}

Phase-locking was assessed using two of the most widely used metrices, namely the phase-locking value (PLV) [6] and pairwise-phase consistency (PPC) [7]. Both were calculated using routines implemented in the FieldTrip toolbox [62]. The 10-min exploration time was divided into non-overlapping 1-s bins and padded to the next power of two. The complex cross-spectrum was computed using a Hann taper with a spectral smoothing of $0.5 \mathrm{~Hz}$.

\section{Phase-amplitude coupling}

Cross-frequency coupling (CFC, [36]) was assessed using the measure of phase-amplitude coupling (PAC), the statistical relationship between the phase of a lowfrequency and the amplitude of a high-frequency component, in a cross-regional analysis [22, 23]. The 10-min recording was split into 1-min bins during which the PAC was calculated using the Modulation Index (MI, $[23,63])$. Briefly, time-series data was first band-pass filtered in the desired frequency range, followed by a Hilbert transform using the MatLab function hilbert which calculates the real and imaginary part of the signal to obtain the instantaneous amplitude and phase at any given time point. Theta phases were binned into eighteen $20^{\circ}$ intervals, and the mean gamma amplitude was calculated in each phase bin. The distribution across bins was assessed using the Kullback-Leibler divergence [64] and normalized between 0 and 1 . The MI is close to zero if the mean gamma amplitude is uniformly distributed over the theta phases and close to one if the mean gamma amplitude is exceptionally higher within one phase bin [23].

\section{Cross-correlation of instantaneous LFP amplitudes}

To determine whether one signal was leading or lagging the other, amplitude cross-correlations of instantaneous amplitudes of LFP oscillations between all brain regions were performed [10]. The 10-min period was divided into 1- $\mathrm{s}$ bins with a 95\% overlap. First, the two signals were band-pass filtered in the respective frequency range; the Hilbert transform was computed using the MatLab function hilbert to calculate the instantaneous amplitude and the envelope of the signal. The mean amplitude was subtracted, and the cross-correlation between the amplitudes of the two signals was calculated with the MatLab function xcorr over lags ranging from -100 to $+100 \mathrm{~ms}$; the lag at which cross-correlation peaked was determined [10]. While lags below $-100 \mathrm{~ms}$ or above $100 \mathrm{~ms}$ would have led to the exclusion of the respective data point [65], no instances of such lags were found in our dataset. To determine if the obtained lags or leads significantly differed from zero, Wilcoxon's signed rank tests were performed.

\section{Granger causality}

Parametric Granger causality (GC) was calculated using the MVGC-toolbox [66]. GC mainly applies to stationary signals which means that the variances are not excessively changing over time [13, 67]. Therefore, the 10 min period was divided into 1-min bins and the in-built trial averaging function was used to calculate $\mathrm{GC}$ in non-overlapping 10-s sections to ensure reasonable stationarity [68-70]. The 1-min bins were used for the analysis of GC over time and then averaged to obtain a GC value for the whole 10-min testing period. Raw LFP data was sampled down to $250 \mathrm{~Hz}$ to ensure a reasonable model order for autoregressive modelling [14, 66, 71]. The model order was obtained using the Bayesian Information Criterion (BIC, [72]) as it was shown to provide the best fit to electrophysiological data [66]. The model order was fixed to 27 across all animals and trials to obtain comparable results [73]. Non-prefiltered data were used because empirical analyses have shown that filtering time-series data increases the VAR model order and leads to high variances making it unsuitable for GC analysis [71]. To obtain GC values for specific frequency bands, we first computed GC up to the Nyquist frequency and then integrated over the desired frequency range [71]. A permutation procedure implemented in the MVGC-toolbox was performed to test the null hypothesis that values obtained by GC estimation occurred by chance [13, 66]. Non-parametric Granger causality (npGC), directed transfer function (DTF) and partial directed coherence (PDC) were calculated using the FieldTrip toolbox [62]. The same temporal configurations were used as described above for parametric GC, and raw LFP data was sampled down to $250 \mathrm{~Hz}$ as well. 
Instead of deriving the noise covariance matrix and transfer function by autoregressive modelling (as done for parametric GC), these were obtained by applying Wilson's spectral matrix factorization to complex Fourier spectra. This non-parametric approach was shown to be better at capturing all spectral features, less errorprone because no model order had to be chosen and computationally faster $[15,35]$.

\section{Spike-phase coupling}

Multi-unit activity was extracted by high-pass filtering the raw signal above $800 \mathrm{~Hz}$ and applying a threshold at 3.5 standard deviations from the mean. Spikes were excluded, if the threshold exceeding was longer than $2 \mathrm{~ms}$, and if spikes occurred within $1 \mathrm{~ms}$ form each other. LFP of the second brain region was filtered between 5 and $12 \mathrm{~Hz}$ using the eegfilt-function of the EEGLAB-toolbox [74]. To account for speed-dependent waveform asymmetry in the theta oscillation, the theta phase was defined by linear interpolation between troughs of consecutive cycles $[75,76]$. Only periods in which the theta amplitude was above 0.25 standard deviations of its mean were included to ensure sufficient theta oscillations and prevent spurious phase determination. The number of spikes was fixed to 1000 for each recording to prevent spuriously high MRL values and fluctuations in the firing rate. Each spike was assigned a theta phase, and the mean resultant vector length (MRL) was calculated as an indicator for the strength of coupling using the CircStat-Toolbox $[2,77]$. The MRL gets close to one when the spikes are concentrated around a certain phase of the theta oscillation and approaches zero when they are uniformly distributed. Additionally, the phase angles of the mean resultant vector were used to quantify the differences in phase angles between genotypes, which were statistically assessed with the Watson-Williams test for two samples [20, 77].

To determine the directionality between multiunit activity and theta oscillations, phase-locking was calculated for 50 different temporal offsets ranging from -100 to +100 $\mathrm{ms}$ in steps of $4 \mathrm{~ms}$. If the MRL peaked at a positive offset, spikes were most strongly locked to the next theta cycle, suggesting that spiking activity drives theta [19]. Wilcoxon's signed rank test was applied to determine if the lag or lead was significantly different from zero.

\section{Statistics}

Genotype-related differences within the same metric and frequency range were assessed by independent-sample $t$ test or, in the case of GC (Fig. 3), by Sidak paired post hoc tests conducted after a significant effect of genotype or interaction in the prior repeated-measures (RM) ANOVA. For circular data (spike and coherence phase angles) the Watson-Williams two-sample test was used to assess genotype-related differences. A $p$ value $<0.05$ was used as an indicator for statistical significance; no further correction for multiple comparisons were applied, given that we aimed to emulate the situation that only a single measure is used to characterize connectivity, and false negatives were to be avoided given the analytical goal of detecting redundancies between metrics. Bivariate correlations were calculated using Spearman's rho. To detect correlations between circular and circular and between circular and linear data, we used circularcircular correlation and circular-linear correlation as implemented in [77]. Variability in the data is displayed as standard error of the mean (SEM) throughout.

\section{Supplementary Information}

The online version contains supplementary material available at https://doi. org/10.1186/s12915-021-00950-4

Additional file 1: Contains four tables, Table S1-S4, that state the results of pairwise Spearman correlations between all metrics analysed in this study for a given connection. The first number in each cell states Spearman's rho, the second number its $p$-value. Significant correlations are highlighted in green. See the 'Info' sheet of the file for further information, including $n$-numbers. Table S1. Correlations of connectivity metrics along the PFC-dHC connection, corresponding to main Figure 5a. Table S2. Correlations of connectivity metrics along the PFC-vHC connection, corresponding to main Figure $5 \mathrm{~b}$. Table S3. Correlations of connectivity metrics along the $\mathrm{VHC}-\mathrm{dHC}$ connection, corresponding to main Figure 6a. Table S4. Average correlations of connectivity metrics along all three connections, corresponding to main Figure 6b. Each cell lists the average Spearman's rho, followed by an indicator of a significant correlation in all three connections (0) or lack of it (1).

\section{Acknowledgements}

We are grateful to Drs. Torfi Sigurdsson (Goethe University Frankfurt) and Lionel Barnett (University of Sussex) for the advice on the analysis procedures, to Dr. Rolf Sprengel (Max-Planck Institute for Medical Research, Heidelberg) for the provision of Gria $1^{-1-}$ mice, and to Stefanie Schulz (UIm University) for the assistance with histology.

\section{Authors' contributions}

DS and DK designed the study and wrote the manuscript which was revised by all authors. DS and SKTK performed the experiments. DS performed the data analysis, supported by AMB. The authors read and approved the final manuscript.

\section{Funding}

This work was funded by the Else Kroener-Fresenius/German Scholars Organization Programme for excellent medical scientists from abroad (GSO/ EKFS 12; to D.K.), the Juniorprofessorship programme of Baden-Württemberg (to D.K.), the DFG (KA 4594/2-1; to D.K.), the Brain and Behaviour Research Foundation (BBR/NARSAD, to D.K.) and the Institute of Applied Physiology of UIm University. Open Access funding enabled and organized by Projekt DEAL.

\section{Availability of data and materials}

The electrophysiological datasets used and analysed in the current study are deposited under https://gin.g-node.org/KaetzelLab/GluA1-KO_LFP_data [78]. All MatLab ${ }^{\circledR}$ analysis scripts are available on https://github.com/KaetzelLab/ LFP_analysis [79].

Ethics approval and consent to participate

All experiments were performed in accordance with the German Animal Rights Law (Tierschutzgesetz) 2013 and were approved by the Federal 
Ethical Review Committee (Regierungsprädsidium Tübingen) of BadenWürttemberg, Germany (licence number TV1399).

\section{Consent for publication}

Not applicable.

\section{Competing interests}

The authors declare that they have no competing interests.

\section{Author details}

${ }^{1}$ Institute of Applied Physiology, Ulm University, Albert-Einstein-Allee 11 89081 Ulm, Germany. ${ }^{2}$ Department of Neuroscience, Johns Hopkins University, Baltimore, USA.

Received: 29 July 2020 Accepted: 5 January 2021

\section{Published online: 08 February 2021}

\section{References}

1. Jones MW, Wilson MA. Theta rhythms coordinate hippocampal-prefrontal interactions in a spatial memory task. PLoS Biol. 2005;3:e402.

2. Sigurdsson T, Stark KL, Karayiorgou M, Gogos JA, Gordon JA. Impaired hippocampal-prefrontal synchrony in a genetic mouse model of schizophrenia. Nature. 2010;464:763-7.

3. Nolte G, Bai O, Wheaton L, Mari Z, Vorbach S, Hallett M. Identifying true brain interaction from EEG data using the imaginary part of coherency. Clin Neurophysiol. 2004;115:2292-307.

4. Stam CJ, Nolte G, Daffertshofer A. Phase lag index: assessment of functional connectivity from multi channel EEG and MEG with diminished bias from common sources. Hum Brain Mapp. 2007;28:1178-93.

5. Vinck M, Oostenveld R, van Wingerden M, Battaglia F, Pennartz CMA. An improved index of phase-synchronization for electrophysiological data in the presence of volume-conduction, noise and sample-size bias. Neurolmage. 2011;55:1548-65.

6. Lachaux J-P, Rodriguez E, Martinerie J, Varela FJ. Measuring phase synchrony in brain signals. Hum Brain Mapp. 1999;8:194-208.

7. Vinck M, van Wingerden M, Womelsdorf T, Fries P, Pennartz CMA. The pairwise phase consistency: a bias-free measure of rhythmic neuronal synchronization. Neurolmage. 2010;51:112-22.

8. Catanese J, Carmichael JE, van der Meer MAA. Low- and high-gamma oscillations deviate in opposite directions from zero-phase synchrony in the limbic corticostriatal loop. J Neurophysiol. 2016;116:5-17.

9. Witham $\mathrm{CL}$, Riddle CN, Baker MR, Baker SN. Contributions of descending and ascending pathways to corticomuscular coherence in humans. J Physiol. 2011;589(Pt 15):3789-800.

10. Adhikari A, Sigurdsson T, Topiwala MA, Gordon JA. Cross-correlation of instantaneous amplitudes of field potential oscillations: a straightforward method to estimate the directionality and lag between brain areas. J Neurosci Methods. 2010;191:191-200.

11. Ding M, Chen Y, Bressler SL. Granger causality: basic theory and application to neuroscience. 2006. https:/arxiv.org/abs/q-bio/0608035v1. Accessed 4 Jul 2020.

12. Bressler SL, Seth AK. Wiener-granger causality: a well established methodology. Neurolmage. 2011;58:323-9.

13. Brovelli A, Ding M, Ledberg A, Chen Y, Nakamura R, Bressler SL. Beta oscillations in a large-scale sensorimotor cortical network: directional influences revealed by Granger causality. Proc Natl Acad Sci. 2004;101:9849-54.

14. Seth AK, Barrett AB, Barnett L. Granger causality analysis in neuroscience and neuroimaging. J Neurosci. 2015;35:3293-7.

15. Dhamala M, Rangarajan G, Ding M. Estimating granger causality from Fourier and Wavelet Transforms of Time Series Data. Phys Rev Lett. 2008; 100:018701.

16. Baccalá LA, Sameshima K. Partial directed coherence: a new concept in neural structure determination. Biol Cybern. 2001;84:463-74.

17. Kaminski MJ, Blinowska KJ. A new method of the description of the information flow in the brain structures. Biol Cybern. 1991;65:203-10.

18. Blinowska KJ. Review of the methods of determination of directed connectivity from multichannel data. Med Biol Eng Comput. 2011;49:521-9.

19. Siapas AG, Lubenov EV, Wilson MA. Prefrontal phase locking to hippocampal theta oscillations. Neuron. 2005;46:141-51.

20. Dickerson DD, Wolff AR, Bilkey DK. Abnormal long-range neural synchrony in a maternal immune activation animal model of schizophrenia. J Neurosci. 2010;30:12424-31.
21. Adhikari A, Topiwala MA, Gordon JA. Synchronized activity between the ventral hippocampus and the medial prefrontal cortex during anxiety. Neuron. 2010;65:257-69.

22. Sirota A, Montgomery S, Fujisawa S, Isomura Y, Zugaro M, Buzsáki G. Entrainment of neocortical neurons and gamma oscillations by the hippocampal theta rhythm. Neuron. 2008;60:683-697.

23. Tort ABL, Kramer MA, Thorn C, Gibson DJ, Kubota Y, Graybiel AM, et al. Dynamic crossfrequency couplings of local field potential oscillations in rat striatum and hippocampus during performance of a T-maze task Proc Natl Acad Sci. 2008;105:20517-22.

24. Zhang X, Zhong W, Brankačk J, Weyer SW, Müller UC, Tort ABL, et al. Impaired theta-gamma coupling in APP-deficient mice. Sci Rep. 2016;6:1-10.

25. Akam T, Kullmann DM. Oscillations and filtering networks support flexible routing of information. Neuron. 2010;67:308-20.

26. Fries P. Rhythms for cognition: communication through coherence. Neuron. 2015;88:220-35

27. Fries P. A mechanism for cognitive dynamics: neuronal communication through neuronal coherence. Trends Cogn Sci. 2005;9:474-80.

28. Womelsdorf T, Schoffelen J-M, Oostenveld R, Singer W, Desimone R, Engel AK, et al. Modulation of neuronal interactions through neuronal synchronization. Science. 2007;316:1609-12.

29. Kajikawa Y, Schroeder CE. How local is the local field potential? Neuron. 2011;72:847-58.

30. Bastos AM, Schoffelen J-M. A tutorial review of functional connectivity analysis methods and their interpretational pitfalls. Front Syst Neurosci. 2016;9. https://doi.org/10.3389/fnsys.2015.00175.

31. Friston KJ. Functional and effective connectivity in neuroimaging: a synthesis. Hum Brain Mapp. 1994;2:56-78.

32. Bolkan SS, Stujenske JM, Parnaudeau S, Spellman TJ, Rauffenbart C, Abbas $\mathrm{Al}$, et al. Thalamic projections sustain prefrontal activity during working memory maintenance. Nat Neurosci. 2017;20:987-96.

33. Likhtik E, Stujenske JM, Topiwala MA, Harris AZ, Gordon JA. Prefrontal entrainment of amygdala activity signals safety in learned fear and innate anxiety. Nat Neurosci. 2014;17:106-13.

34. Stujenske JM, Likhtik E, Topiwala MA, Gordon JA. Fear and safety engage competing patterns of theta-gamma coupling in the sasolateral amygdala. Neuron. 2014;83:919-33.

35. Dhamala M, Rangarajan G, Ding M. Analyzing information flow in brain networks with nonparametric Granger causality. Neurolmage. 2008;41:354-62.

36. Canolty RT, Knight RT. The functional role of cross-frequency coupling. Trends Cogn Sci. 2010;14:506-15.

37. Colgin LL, Denninger T, Fyhn M, Hafting T, Bonnevie T, Jensen $\mathrm{O}$, et al. Frequency of gamma oscillations routes flow of information in the hippocampus. Nature. 2009;462:353-7.

38. Tort ABL, Komorowski RW, Manns JR, Kopell NJ, Eichenbaum H. Thetagamma coupling increases during the learning of item-context associations. Proc Natl Acad Sci. 2009;106:20942-7.

39. Axmacher N, Henseler MM, Jensen O, Weinreich I, Elger CE, Fell J. Crossfrequency coupling supports multi-item working memory in the human hippocampus. Proc Natl Acad Sci. 2010;107:3228-33.

40. Nandi B, Swiatek $P$, Kocsis B, Ding M. Inferring the direction of rhythmic neural transmission via inter-regional phase-amplitude coupling (ir-PAC). Sci Rep. 2019;9:1-13.

41. Lisman JE, Jensen O. The theta-gamma neural code. Neuron. 2013;77:1002-16.

42. Frank LM, Brown EN, Wilson MA. A comparison of the firing properties of putative excitatory and inhibitory neurons from CA1 and the entorhinal cortex. J Neurophysiol. 2001;86:2029-40.

43. Paré D, Gaudreau H. Projection cells and interneurons of the lateral and basolateral amygdala: distinct firing patterns and differential relation to theta and delta rhythms in conscious cats. J Neurosci. 1996;16:3334-50.

44. Benchenane K, Peyrache A, Khamassi M, Tierney PL, Gioanni Y, Battaglia FP, et al. Coherent theta oscillations and reorganization of spike timing in the hippocampal-prefrontal network upon learning. Neuron. 2010;66:921-36.

45. Tan Z, Robinson HL, Yin D-M, Liu Y, Liu F, Wang H, et al. Dynamic ErbB4 activity in hippocampal-prefrontal synchrony and top-down attention in rodents. Neuron. 2018;98:380-393.e4.

46. Parnaudeau S, O Neill P-K, Bolkan SS, Ward RD, Abbas Al, Roth BL, et al. Inhibition of mediodorsal thalamus disrupts thalamofrontal connectivity and cognition. Neuron. 2013;77:1151-1162.

47. Seidenbecher T, Laxmi TR, Stork O, Pape HC. Amygdalar and hippocampal theta rhythm synchronization during fear memory retrieval. Science. 2003; 301:846-50. 
48. Bygrave AM, Jahans-Price T, Wolff AR, Sprengel R, Kullmann DM, Bannerman DM, et al. Hippocampal-prefrontal coherence mediates working memory and selective attention at distinct frequency bands and provides a causal link between schizophrenia and its risk gene GRIA1. Transl Psychiatry. 2019; 9:142

49. Barkus C, Sanderson DJ, Rawlins JNP, Walton ME, Harrison PJ, Bannerman DM. What causes aberrant salience in schizophrenia? A role for impaired short-term habituation and the GRIA1 (GluA1) AMPA receptor subunit. Mol Psychiatry. 2014;19:1060-70.

50. Fraschini M, La Cava SM, Didaci L, Barberini L. On the variability of functional connectivity and network measures in source-reconstructed EEG time-series. arXiv.org. 2020. https://arxiv.org/abs/2007.05395v1. Accessed 16 Jul 2020.

51. Takata N, Yoshida K, Komaki Y, Xu M, Sakai Y, Hikishima K, et al. Optogenetic activation of CA1 pyramidal neurons at the dorsal and ventral hippocampus evokes distinct brain-wide responses revealed by mouse fMRI. PLoS One. 2015;10:e0121417.

52. Bienkowski MS, Bowman I, Song MY, Gou L, Ard T, Cotter K, et al. Integration of gene expression and brain-wide connectivity reveals the multiscale organization of mouse hippocampal networks. Nat Neurosci. 2018;21:1628-43.

53. Spellman T, Rigotti M, Ahmari SE, Fusi S, Gogos JA, Gordon JA. Hippocampal-prefrontal input supports spatial encoding in working memory. Nature. 2015;522:309-14.

54. Jay TM, Glowinski J, Thierry A-M. Selectivity of the hippocampal projection to the prelimbic area of the prefrontal cortex in the rat. Brain Res. 1989;505: 337-40.

55. Swanson LW. A direct projection from Ammon's horn to prefrontal cortex in the rat. Brain Res. 1981;217:150-4.

56. Vertes RP. Differential projections of the infralimbic and prelimbic cortex in the rat. Synapse. 2004;51:32-58.

57. Vertes RP. Interactions among the medial prefrontal cortex, hippocampus and midline thalamus in emotional and cognitive processing in the rat. Neuroscience. 2006;142:1-20.

58. Barnett L, Barrett AB, Seth AK. Solved problems for Granger causality in neuroscience: a response to stokes and Purdon. Neurolmage. 2018;178:7448.

59. Herreras O. Local field potentials: myths and misunderstandings. Front Neural Circuits. 2016;10. https://doi.org/10.3389/fncir.2016.00101.

60. Zamanillo D, Sprengel R, Hvalby O, Jensen V, Burnashev N, Rozov A, et al. Importance of AMPA receptors for hippocampal synaptic plasticity but not for spatial learning. Science. 1999;284:1805-11.

61. Bokil H, Andrews P, Kulkarni JE, Mehta S, Mitra PP. Chronux: a platform for analyzing neural signals. J Neurosci Methods. 2010;192:146-51.

62. Oostenveld R, Fries P, Maris E, Schoffelen J-M. FieldTrip: open source software for advanced analysis of MEG, EEG, and invasive electrophysiological data. Comput Intell Neurosci. 2010;2011. https://doi.org/ 10.1155/2011/156869.

63. Voloh B, Valiante TA, Everling S, Womelsdorf T. Theta-gamma coordination between anterior cingulate and prefrontal cortex indexes correct attention shifts. Proc Natl Acad Sci. 2015. https://doi.org/10.1073/pnas.1500438112.

64. Kullback S, Leibler RA. On information and sufficiency. Ann Math Stat. 1951; 22:79-86.

65. Ishino S, Takahashi S, Ogawa M, Sakurai Y. Hippocampal-prefrontal theta phase synchrony in planning of multi-step actions based on memory retrieval. Eur J Neurosci. 2017;45:1313-24.

66. Barnett $L$, Seth AK. The MVGC multivariate granger causality toolbox: a new approach to Granger-causal inference. J Neurosci Methods. 2014;223:50-68.

67. Geweke JF. Measures of conditional linear dependence and feedback between time series. J Am Stat Assoc. 1984;79:907-15.

68. Florian G, Pfurtscheller G. Dynamic spectral analysis of event-related EEG data. Electroencephalogr Clin Neurophysiol. 1995;95:393-6.

69. Hesse W, Möller E, Arnold M, Schack B. The use of time-variant EEG granger causality for inspecting directed interdependencies of neural assemblies. J Neurosci Methods. 2003;124:27-44.

70. Jeong J, Gore JC, Peterson BS. A method for determinism in short time series, and its application to stationary EEG. IEEE Trans Biomed Eng. 2002;49: 1374-9.

71. Barnett L, Seth AK. Behaviour of Granger causality under filtering: theoretical invariance and practical application. J Neurosci Methods. 2011;201:404-19.

72. Schwarz G. Estimating the dimension of a model. Ann Stat. 1978;6:461-4.
73. Cohen MX. Analyzing neural time series data: theory and practice. The MIT Press; 2014. https://mitpress.mit.edu/books/analyzing-neural-time-series-data. Accessed 4 Jul 2020.

74. Delorme A, Makeig S. EEGLAB: an open source toolbox for analysis of single-trial EEG dynamics including independent component analysis. J Neurosci Methods. 2004;134:9-21.

75. Buzsáki G, Rappelsberger P, Kellényi L. Depth profiles of hippocampal rhythmic slow activity ('theta rhythm') depend on behaviour. Electroencephalogr Clin Neurophysiol. 1985;61:77-88.

76. McClain K, Tingley D, Heeger DJ, Buzsáki G. Position-theta-phase model of hippocampal place cell activity applied to quantification of running speed modulation of firing rate. Proc Natl Acad Sci. 2019;116:27035-42.

77. Berens P. CircStat: a MATLAB toolbox for circular statistics. J Stat Softw. 2009; $31: 1-21$.

78. Strahnen D, Kapanaiah S, Bygrave AM, Kätzel D. KaetzelLab/GluA1-KO_LFP_ data. Ger Neuroinformatics Node. 2020. doi:https://doi.org/10.12751/g-node. 3lvs3q.

79. Strahnen D, Kätzel D. KaetzelLab/LFP_analysis. Github. 2020. https://doi.org/ 10.5281/zenodo.4384980.

\section{Publisher's Note}

Springer Nature remains neutral with regard to jurisdictional claims in published maps and institutional affiliations.
Ready to submit your research? Choose BMC and benefit from:

- fast, convenient online submission

- thorough peer review by experienced researchers in your field

- rapid publication on acceptance

- support for research data, including large and complex data types

- gold Open Access which fosters wider collaboration and increased citations

- maximum visibility for your research: over $100 \mathrm{M}$ website views per year

At BMC, research is always in progress.

Learn more biomedcentral.com/submissions 